\title{
XXVIII.
}

(Aus dem Istituto dei Rachitici in Mailand. Vorstand: Prof. Dr. B. Galeazzi.)

\section{Zur Deutung der umschriebenen jugendlichen Formen der ,tumorbildenden Ostitis fibrosa (v. Recklinghausensche Krankheit)".}

Von

Dr. Achille AImerini, Assistenzarzt.

(Hierzu Tafel XVII und 10 Textfiguren.)

Aus einigen Beobachtungen v. Recklinghausens, welche er in seiner ausgezeichneten Monographie 1891 darstellte, und aus anderen später von verschiedenen Autoren veröffentlichten ähnlichen Beobachtungen ging das Bild einer besonderen Erkrankung des Knochensystems hervor, welche abwechselnd als "v. Recklinghausensche Knochenkrankheit" und als "Ostitis (Osteomyelitis) fibrosa" bezeichnet wurde. Die Richtigkeit der gleichgiltigen und synonymen Auwendung dieser beiden Namen ist sehr fraglich.

Bei der Beschreibung der Fälle, welche die typischen Exemplare der erwähnten Osteopathie biłsen, bezeichnet Verf. den Prozess einmal (Fall V) als "verbreitete deformierende Ostitis mit Tumoren", während er ihn bei einer anderen Beobachtung (Fall VII) mit der Benemnung "fibröse Ostitis, multiple Osteosarkome" zusammenfasst und ihn noch anderswo "tumorbildende Ostitis fibrosa" nennt. Anatomisch ist nämlich die Krankheit nicht durch die einfache fibröse Entartung des Knochenmarkes charakterisiert, welche einen sehr gewöhnlichen Prozess darstellt und auch bei mehreren anderen Erkrankuugen des Knochensystems beobachtet wurde, sondern durch die Vereinigung eines mehr oder minder diffus in Form von kleinen, myeloiden Sarkomen und von Fibromen (Fibrosarkomen) verbreiteten geschwulstartigen, stellenweise von cystischer Entartung befallenen Elementes, mit einem diffusen Involutionsprozesse des 
Knochengewebes, wahrscheinlich entzündlicher Natur. Dies geht anss der Unwandlung des normalen adipösen oder lymphatischen Knochenmarkes in ein faseriges Bindegewebe (Fasermark) hervor, welche von Erscheinungen der Halisteresis, der lakunären Resorption und einer unvollstăndigen Knochenueubildung (Bildung von osteoidem Gewebe) begleitet ist.

Die erwähnte Vereinigung stellt nicht ein sonderbares zufälliges, für v. Recklinghausens Fälle charakteristisches Zusammentreffen dar, sondern wurde mehrfach bei ähnlichen Krankheitsformen beobachtet, weshalb wir sie als einen wesentlichen Teil des Krankheitsbildes ansehen dürfen und müssen, wenn wir die r. Recklinghausensche Osteopathie als eine selbständige Krankheitsform ansprechen wollen. Sonst müsste man sie mit der Pagetschen Krankheit (chronische deformierende hypertrophisehe Osteomalacie) zusammentun, von welcher sie sich klinisch mur durch die ausgesprochene Neigung zu spontanen Frakturen und durch eine geringere Gutartigkeit unterscheidet. Diese Eigentümlichkeiten scheinen gerade mit der Anwesenheit der Tumoren und besonders der Riesenzellensarkome zusammenzuhängen.

Während einige Autoren, sich auf die Aelmlichkeit der diffusen histologischen Veränderungen (Osteomyelitis fibrosa) und der gröberen Slielettveränderungen (Verdickungen und Verkrümmungen) bei den beiden Formen und auf das genaue Entsprechen des Sitzes und der Dauer der beiden Erkrankungen stïtzend, die Identität der beiden Krankheitsformen vertreten, glauben wir dieselben rationell unterscheiden zu können. Die Pagetsche Form and die v. Recklinghausensche typische Form sind beide diftuse deformierende Skeletterkrankungen, welche sich im späten Alter auf einem gleichen osteomalacischen Grunde entwickeln; während aber erstere eine einfache fibröse osteoplastische Osteomyelitis darstellt, ist letztere eine fibröse osteoplastische Osteomyelitis mit Tumorbildung. Deshalb gehören nach meiner Ansicht die Fälle von Pagetscher Ostitis, bei welcher multiple Sarkome beobachtet wurden, in die Gruppe der v. Recklinghausenschen Osteopathie hinein.

Es wird auch heutzutage noch viel über die Frage diskutiert, wie die Tumorbildung bei v. Recklinghausenscher Knochenkrankheit gedeutet werden muss, d. h. ob die Neubildungen als Blastome oder als Geschwülste in engem Sinne angesprochen oder als entzündliche Neoplasien betrachtet werden sollen. Die Lösung dieser Frage kann man weniger von den histologischen Untersuchungen als vielmehr von den klinischen Beobachtungen erwarten. Die mikroskopischen Untersuchungen haben bis jetzt keine wesentlichen morphologischen Unterschiede zwischen den myeloiden Sarkomen und den rötlichbraunen, an Riesenzellen reicheu, kleinen GeschwüIsten enthüllt, welche in einem der Fälle von v. Recklinghausen und in verschiedenen anderen ähnichen, später veröffentlichten Fällen 
beobachtet wurden; in anderen Fällen hat die mikroskopische Untersuchung: bei den endoostealen bindegewebigen Neubildungen der tumorbildenden Ostitis fibrosa das ausgesprochene Aussehen der Fibrome und der Fibrosarkome gezeigt. Weiteres können wir von der histologischen Forschung nicht verlangen.

Dagegen verfügt die Klinik heutzutage über eine ziemlich reiche Kasuistik, welche genügt, um durch eine parallele Kritik der Krankengeschichten und der Befunde zu einigen Schlussfolgerungen kommen zu können.

Die Frage nach der neoplastischen oder entzündlichen Entstehung stellt sich mit einer besonderen Klarheit und vielleicht in einer einfacheren Weise in bezug auf die jugendliche umschriebene Form der uns hier interessierenden Osteopathie, von welcher ich hier zwei an sich ziemlich interessante Beispiele mitteilen und illustrieren kann. Bevor ich zur Beschreibung derselben schreite, erscheint es mir angezeigt, auf Grund der vorliegenden Literatur die wichtigsten und ausgesprochensten Kennzeichen dieser Form, im Vergleich zu derjenigen der typischen Form, und die hauptsächlichen Deutungen, welche von den verschiedenen Autoren der ersteren Form gegeben wurden, zu erwähnen.

Als Paradigmata des Krankheitsbildes der tumorbildenden Ostitis fibrosa kann man die von v. Recklinghausen erwähnten Fälle V und VII betrachten. Bei denselben, welche beide ältere Patienten betreffen, handelte es sich um ein an zahlreichen Abschnitten des Skeletts entwickeltes Knochenleiden, welches darin die oben erwähnten Veräuderungen bedingte. Während jedoch im Fall VII die geschwulstartigen Bildungen ausschliesslich aus Riesenzellensarkomen bestanden, wurde in Fall V kein Sarkom, sondern nur Cystofibrome nachgewiesen.

Wenn wir nun die seitdem erschienene ziemlich reiche Literatur durchmustern, finden wir eine ganze Reihe von Beobachtungen, deren klinisches und anatomisches Bild, im grossen und ganzen genommen, mit v. Recklinghausens Schilderung übereinstimmt. l)agegen wurden in neuerer Zeit von verschiedenen Autoren mehrere Fälle als Ostitis fibrosa angesprochen, deren Einreihung ins Gebiet der v. Recklinghausenschen Osteopathie dasselbe seiner früheren Homogenität beraubt. Es erscheint mir zweckmässig, die bisher beschriebenen Fälle in zwei verschiedene Klassen: ju gendliche Formen ${ }^{1}$ ) und Formen des erwachsenen und des senilen

1) v. Recklinghausen schliesst überhaupt nicht aus, dass auch in früherer Lebenszeit fibröse ostitische Erkrankungen das Skelett und besonders einzelne Skelettabschnitte anzugreifen mögen. Er meint sogar als eine solche die im Falle von Bilton-Pollard vorhandene andeuten zu dürfen (hyperplastische Osteome, vermutlich traumatischen Ursprungs, in der gebogenen Mitte der Tibia und Fibula eines 5jährigen nicht rachitischen Kindes). v. Recklinghausen, Die fibröse oder deformierende Ostitis. Berlin, 1891. S. 72. 
Alters einzuteilen, welche wieder in diftuse und umschriebene Formen eingeteilt werden können.

Un ihre Vergleichung zu erleichtern, habe ich sämtliche mir bekannten Beobachtungen, denen ein mikroanatomischer Befund beigegeben ist, in folgender Tabelle (S. 394 ff.) kurz zusammengestellt. Als Altersgrenze für die Verteilung der Fälle in den genannten Kategorien bestimme ich das 23. Lebensjahr, welches bekanntlich in der Regel dem Abschlusse des gesamten Skelettwachstums entspricht. In der Tabelle habe ich anch einzelne Fälle mitgezählt, deren anatomisch - pathologische Vorgänge anders als tumorbildende Ostitis fibrosa bezeichnet würden, indem selbst die Beobachter derselben die Aehnlichkeit der klinischen Erscheinungen und der anatomischen Veränderungen mit denjenigen der tumorbildenden Ostitis fibrosa, oder vielmehr die Gleichwertigkeit des Prozesses ausdrücklich zugeben.

Jenseits des 23. Lebensjahres finden wir also ein einziges Beispiel von umschriebener tumorbildender Ostitis fibrosa (Beobachtungen von BötticherMönckeberg). Der Fall ron Schmieden (Erkrankung des Unterschenkels bei einer 58jährigen Frau, welchen ich als zweifelhaft bezeichnet habe) stellt höchstwahrscheinlich eine Iokalisierte $\mathrm{Pagetsche}$ Krankheit und keine tumorbildende Ostitis fibrosa dar. Dasselbe kann man für v. Recklingbausens Fälle I, III und IV annehmen. Ebenso möchte ich eine der Beobachtungen von Pfeiffer nicht in Betracht ziehen, weil bei derselben (Cyste im unteren Drittel des linken Oberschenkels bei einer 41 jährigen Frau, ohne Knochenauftreibung noch Verbiegung), obwohl der histologische Befund auf eine Osteomyelitis fibrosa in der. Umgebung der Cyste hinweist, die übrigen klinischen und anatomischen Zeichen der v. Recklinghausenschen Osteopathie fehlen (vgl. Tab. I).

Im Gegensatze zu dem, was im erwachsenen und senilen Alter beobachtet wird, wo die Erkrankung sich diffus oder in verschiedenen Knochen verstrent zeigt, gehört in der Kindheit und im jugendlichen Alter die grosse Mehrzahl der Fälle einer örtlichen Spielart der Krankbeit an.

Sämtliche Abweichungen letzterer von oben geschildertem Typus sollen hier kurz hervorgehoben werden:

1. Die Erkrankung beginnt und verläuft in der Kindheit oder in der Jugend, jedenfalls in der Epoche des Wachtumsabschlusses der befallenen Knochen.

2. Sie ist streng auf einen einzigen, höchstens auf zwei aneinanderliegende Skelettabschnitte beschränkt; nur selten haudelt es sich um svmmetrische Herde.

3. Während bei der echten v. Recklinghausenschen Krankheit die Veränderungen vorwiegend die Diaphysen in ihrer Mitte befallen, lokalisieren sie sich dagegen bei der jugendlichen umschriebenen Form mit Vorliebe in den paraepiphysären Gegenden, also in den Metapbysen. 
4. Abgesehen von einem Falle von Pfeiffer, wird beim Bericht über den mikroskopischen Befund das Vorhandensein von Riesenzellensarkomen nicht erwähnt, oder sogar die Abwesenheit derselben ausdrücklich betont; höchstens wurden im krankhaften Gewebe einzelne zerstreute Riesenzellen nachgewiesen.

5. Dagegen wurden bei den Fällen von Küster, Bockenheimer, Tietze, und bei einem von Pfeiffer, inmitten der faserigen endostalen Neubildung Knorpelinseln gefpnden.

An und für sich hätte die Beschränkung, d. b. das Umschriebensein der Veränderungen, keine besonders grosse Wichtigkeit; man dürfte nur meinen, dass solche örtlichen Knochenerkrankungen als initiale Erscheinungen einer diffusen Skeletterkrankung anzusehen sind. Dass bei der jugendlichen Form der tumorbildenden Ostitis fibrosa neue aufeinanderfolgende Herde entstehen können, wurde von v. Haberer mit Sicherheit bewiesen. Die sehr ausführlich beschriebene Beobachtung dieses Autors betrifft einen der seltenen Fälle von verbreiteter jugendlicher Ostitis fibrosa, welcher sich, im Gegensatz zu dem gewöhnlichen anatomischen Verhalten der umschriebenen Form, durch ein reichliches Vorkommen und eine erhebliche Entwicklung von Riesenzellensarkomen auszeichnet.

Nun ist ja gerade das Zusammentreffen dieser beiden Erscheinungen: begrenzter Sitz der Erkrankung und nahezu regelmässige Abwesenheit von riesenzellensarkomartigen Bildungen, das was uns bei der jugendlichen Form am meisten bemerkenswert erscheint. Wir könnten überhaupt nicht diese gleichzeitigen Umstände miteinander in Zusammenhang bringen, ohne solchen Riesenzellensarkomen den Wert und die Bedeutung wirklicher Tumoren anzuerkennen. Darüber erregt uns doch die hohe Autorität jener Forscher Bedenken, welche solche Bedeutung angezweifelt haben oder sogar die entzündliche Entstehung der riesenzellensarkomartigen Bildung der Ostitis fibrosa vertreten haben.

In allererster Linie sei hier v. Recklinghausen genannt, welcher zwar kein kategorisches Urteil fällt, jedoch den Ursprung und den eigentümlichen Sitz solcher Geschwülste von gewissen mechanischen und zirkulatorischen Momenten abhängen lässt.

Rehn scheint sogar die Heilung der Pseudomyelome und ihre Substitution durch fibrös-osteoide Gewebsmassen beobachtet zu haben; des weiteren setzt er den langsameren, ziemlich gutartigen Verlauf der Krankheit dem gewöhnlichen Verhalten der echten Sarkome entgegen.

Heinecke stellt die Frage, ob die Entwicklung von Sarkomen nicht zuweilen durch irgend einen auf das sonst veränderte Knochen- und Markgewebe von den Frakturen ausgeübten Reiz bedingt werden kann.

Gaugele stellt in einer kürzlich im Archiv für klinische Chirurgie Zeitschrift für Krebsforschung. 7. Bd. 2. Heft. 
Tabelle I. Tumorbildende Ostitis

\begin{tabular}{|c|c|c|c|c|c|}
\hline \multirow{2}{*}{ Autoren } & \multirow{2}{*}{ 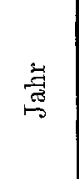 } & \multicolumn{4}{|c|}{$\begin{array}{l}\text { Umsehriebene Formen (ein einziger oder zwei benachbarte bzw. } \\
\text { symmetrische Skelettabschnitte befallen) }\end{array}$} \\
\hline & & $\begin{array}{c}\text { Alter, } \\
\text { Ge- } \\
\text { schlecht }\end{array}$ & Sitz & Anatomischer Befund & $\begin{array}{l}\text { Verlauf } \\
\text { Ausgang }\end{array}$ \\
\hline Küster & 1897 & 17 우 & $\begin{array}{l}\text { Oberes Ende des } \\
\text { rechten Femurs }\end{array}$ & $\begin{array}{l}\text { Fibröse Massen mit kleinen } \\
\text { Cysten; Knochenneubildung; } \\
\text { knorpelige Massen }\end{array}$ & $\begin{array}{l}\text { Tod (Broncho- } \\
\text { pneumonie } \\
\text { infolg: Aether- } \\
\text { narkose }\end{array}$ \\
\hline Rehn & 1904 & 一 & - & 一 & - \\
\hline Heinecke & 1905 & - & - & - & - \\
\hline v. Brunn & 1905 & 10 우 & $\left\{\begin{array}{l}\text { Beide Oberschen- } \\
\text { kel (ober. Ende) }\end{array}\right.$ & $\begin{array}{l}\text { Faserige Umwandlung des Mar- } \\
\text { kes; osteoides Gewebe }\end{array}$ & - \\
\hline v. Brunn & 1906 & 12 む & $\begin{array}{l}\text { Diaphyse des } 1 . \\
\text { linken Humerus }\end{array}$ & $\begin{array}{l}\text { Knochenhöhle, enthaltend } \\
\text { weiches bomogenes Gewebe } \\
\text { (Bindegewebe). Faserige Um- } \\
\text { wandlung des Markes }\end{array}$ & $\begin{array}{l}\text { Heilung dureh } \\
\text { chirurgische } \\
\text { Behandlug }\end{array}$ \\
\hline Glimm & 1905 & 12 \% & $\begin{array}{l}\text { Oberes Ende des } \\
\text { 1. Oberschenkels }\end{array}$ & $\begin{array}{l}\text { Subtroch. - Cyste. Fibröse Um- } \\
\text { wandlung d. Markes; osteoides } \\
\text { Gewebe. }\end{array}$ & - \\
\hline Bockenheimer & 1906 & 17 ? & $\begin{array}{l}\text { Oberes Ende des } \\
\text { r. Oberschenkels, } \\
\text { J. Unterschenkel }\end{array}$ & $\begin{array}{l}\text { Grosse Cyste. Markfibrose; } \\
\text { osteoide Knochenneubildung. } \\
\text { Knorpel }\end{array}$ & Tod (Shok) \\
\hline Tietze & 1906 & 18 ? & $\begin{array}{l}\text { Oberes Ende u. } \\
\text { Diaphyse des 1. } \\
\text { Oberschenkels; } \\
\text { I. Unterschenkel }\end{array}$ & $\begin{array}{l}\text { Cysten. Fibröse Gewebsmassen } \\
\text { mit Knorpelinseln }\end{array}$ & - \\
\hline v. Haberer & $\begin{array}{c}1904 \\
\text { u. } \\
1907\end{array}$ & - & - & -- & - \\
\hline Pfeiffer & 1907 & $21 / 2 \%$ & $\begin{array}{l}\text { Beide Oberschen- } \\
\text { kel (untere Meta- } \\
\text { physen) }\end{array}$ & $\begin{array}{l}\text { Fasermark; einzelne Riesen- } \\
\text { zellen } \\
\text { Fasermark; demFaserknorpel }\end{array}$ & - \\
\hline Pfeiffer & 1907 & 22 우 & $\begin{array}{l}\text { Mittleres Drittel } \\
\text { des recht. Ober- } \\
\text { sehenkels }\end{array}$ & $\begin{array}{c}\text { ähnliches Gewebe. Ein- } \\
\text { zelne Riesenzellen. Cyste. } \\
\text { Cyste = Fasermark; entzünd- }\end{array}$ & Tod (Lungen- \\
\hline Pfeiffer & 1907 & 20 오 & $\begin{array}{l}\text { Unteres Drittel d. } \\
\text { r. Oberschenkels }\end{array} \mid$ & $\begin{array}{l}\text { lich infiltriertes Riesenzellen- } \\
\text { sarkom }\end{array}$ & tuberkulose) \\
\hline
\end{tabular}

erschienenen Arbeit ${ }^{1}$ ) die Meinung von Lubarsch dar, welcher seinen Fall histologisch untersucht hat. Dieser hervorragende Pathologe kommt,

1) Archir f, klin. Chir. Bd. 83. S. 953. 
fibrosa. Jugendliche Formen.

\begin{tabular}{|c|c|c|c|c}
\hline \multicolumn{2}{|c|}{ Nultiple bzw. verbreitete Formen (mehrere entfernte Skelett- } & \\
abschnitte befallen)
\end{tabular}

indem er sich besonders auf den Reichtum dieser Geschwülste an Pigment und auf feine morphologiscbe Differenzen zwischen den Elementen derselben und denjenigen der Epulis und der echten Riesenzellensarkome der Tibia und des Femurs stützt, zu folgender Schlussfolgerung: „Es hande]t 
Tabelle I. Tumorbildende 0stitis

\begin{tabular}{|c|c|c|c|c|c|}
\hline \multirow{2}{*}{ Autoren } & \multirow{2}{*}{ 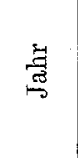 } & \multicolumn{4}{|c|}{ Umsehriebene Formen } \\
\hline & & $\begin{array}{c}\text { Alter, } \\
\text { Ge- } \\
\text { sehlecht }\end{array}$ & Sitz & Anatomischer Befund & $\begin{array}{l}\text { Verlauf, } \\
\text { Ausgang }\end{array}$ \\
\hline v.Recklinghausen & 1891 & - & $\longrightarrow$ & - & - \\
\hline v. Recklinghausen & 1891 & - & - & - & - \\
\hline Hirschberg & 1889 & - & - & - & - \\
\hline Schönenberger & 1901 & - & 一 & - & - \\
\hline Hart & 1904 & - & - & - & - \\
\hline Mönckeberg & 1904 & - & 一 & - & - \\
\hline $\begin{array}{l}\text { Bötticher- } \\
\text { Mönckeberg }\end{array}$ & 1904 & 25 운 & $\begin{array}{c}\text { Oberes Ende } \\
\text { des r. Ober- } \\
\text { armknochens }\end{array}$ & $\begin{array}{l}\text { Multilokuläre Cyste; faserige } \\
\text { Umwandlung des Markes; } \\
\text { riesenzellensarkomartig.Massen }\end{array}$ & $\begin{array}{l}\text { Heilung durch } \\
\text { Resek tion }\end{array}$ \\
\hline Lissauer & 1905 & - & - & - & - \\
\hline Katholicky & 1905 & - & - & - & - \\
\hline Schmieden & 1905 & 58 ㅇ. & $\begin{array}{l}\text { L. Unterschen- } \\
\text { kel in toto }\end{array}$ & $\begin{array}{l}\text { Fasermark (Osteomyelitis } \\
\text { fibrosa) }\end{array}$ & Amputation \\
\hline Pfeiffer & 1907 & 419 & $\begin{array}{l}\text { Unter. Drittel } \\
\text { des l. Femurs }\end{array}$ & $\begin{array}{l}\text { Cyste; weder Verdickung noch } \\
\text { Verbiegung des Knochens; } \\
\text { Osteomyelitis fibrosa; keine } \\
\text { freien Riesenzellen im Binde- } \\
\text { gewebe }\end{array}$ & - \\
\hline Gaugele & 1907 & 一 & 一 & - & - \\
\hline
\end{tabular}

sich in der Hauptsache darum, dass die immer reichlicher auftretenden Osteoblasten, nachdem sie ihr Zerstörungswerk an den Spongiosa- und Kortikalis-Bälkchen vollendet haben, sich zusammenschliessen und wahrscheinlich auch den Reiz der fortwährend hier stattfindenden Blutungen vermehren und als Phagozyten sich mit Pigment beladen.

So erklärt sich ihre Lokalisation an den Frakturen, d. h. den dünnsten, am stärksten eingeschmolzenen Knochenstellen."

Als Beweisgründe der nicht blastomatösen Natur des Riesenzellensarkoms bei Ostitis fibrosa erwähnt ausserdem Gaugele folgende Momente:

a) Ibre sicher nicht auf einer Metastasierung beruhende Multiplizität.

b) Das Ansbleiben eines Einflusses auf das Allgemeinbefinden des Patienten. 
fibrosa. Spätere Formen.

\begin{tabular}{|c|c|c|c|c|}
\hline \multicolumn{3}{|c|}{ Multiple bzw. verbreitete Formen } & \multirow{2}{*}{$\begin{array}{l}\text { Verlauf, } \\
\text { Ausgang }\end{array}$} & \multirow{2}{*}{ Bemerkungen } \\
\hline $\begin{array}{c}\text { Alter, } \\
\text { Ge- } \\
\text { schlecht }\end{array}$ & Sitz & Anatomischer Befund & & \\
\hline $40 \frac{1}{\partial}$ & Verbreitete Erkr. & Cysten; Osteomyelitis fibrosa; & Tod(Marasm.,Ver- & Fall VII \\
\hline 66 우 & dgl. & $\begin{array}{l}\text { Riesenzellensark ome } \\
\text { Cystofibromen; Osteomyelitis } \\
\text { fibrosa. }\end{array}$ & $\begin{array}{l}\text { lautsdauer: 1/2d, } \\
\text { Tod (Pneumonie) }\end{array}$ & Fall V \\
\hline 35 운 & $\begin{array}{l}\text { Oberarm, Femur, } \\
\text { Unterschenkel }\end{array}$ & $\begin{array}{l}\text { Cysten: Osteomalacie; Riesen- } \\
\text { zellensarkome }\end{array}$ & Tod (Marasmus) & - \\
\hline 33 우 & $\begin{array}{l}\text { Rippen, Becken, } \\
\text { l. Humerus, Fe- } \\
\text { mora }\end{array}$ & $\begin{array}{l}\text { Fasermark; Riesenzellensar- } \\
\text { kome }\end{array}$ & $\begin{array}{l}\text { Tod (1 Jahr Ver- } \\
\text { lauf) }\end{array}$ & $\begin{array}{l}\text { Einsetzen der Er- } \\
\text { krankg. währ. d. } \\
\text { Schwangerschaft }\end{array}$ \\
\hline 78 오 & $\begin{array}{l}\text { Verbreitete } \\
\text { Knochenerkrankg. }\end{array}$ & $\begin{array}{l}\text { Cysten; Osteomalacie; multiple } \\
\text { Riesenzellensarkome }\end{array}$ & Tod (Pneumonie) & - \\
\hline 55 우 & dgl. & $\begin{array}{l}\text { Cysten; Fibrome; Riesenzellen- } \\
\text { sarkome }\end{array}$ & Tod (Marasmus) & -- \\
\hline - & - & - & - & - \\
\hline 36 ち & $\begin{array}{l}\text { Verbreitete } \\
\text { Knochenerkrankg. }\end{array}$ & $\begin{array}{l}\text { Fasermark; Knochenschwund; } \\
\text { Myeloid-Sarkom am Finger }\end{array}$ & $\begin{array}{l}\text { Tod (Verlaufs- } \\
\text { dauer } 11 \text { J.) }\end{array}$ & $\begin{array}{l}\text { Partieller Be- } \\
\text { fund }\end{array}$ \\
\hline 30 电. & dgl. & $\begin{array}{l}\text { Osteomalacie; Knochenneubildung; } \\
\text { kernreiches osteoides Gewebe }\end{array}$ & $\begin{array}{l}\text { Tod (Verlaufs- } \\
\text { dauer } 7 \mathrm{~J} .)\end{array}$ & \\
\hline - & 一 & - & - & $\begin{array}{l}\text { (umschriebene } \\
\text { Pagets Form?) }\end{array}$ \\
\hline - & - & & & - \\
\hline 36 오 & $\begin{array}{l}\text { Verbreitete } \\
\text { Knochenerkrankg. }\end{array}$ & $\begin{array}{l}\text { Cysten; fibröse Umwandlung des } \\
\text { Markes; riesenzellensarkom- } \\
\text { artige, rotbraune Tumormassen. }\end{array}$ & $\begin{array}{l}\text { Tod (Prelonephri- } \\
\text { tis; Urämie) }\end{array}$ & - \\
\hline
\end{tabular}

c) Die geringe Anschwellung der befallenen Knochenabschnitte.

d) Die stetig scharfe Abgrenzung der Geschwulst gegen die Weichteile der Umgebung, welche dabei nicht in Mitleidenschaft gezogen sind.

Aus diesen Eigentümlichkeiten tritt eine unverkennbare, durchaus besondere Physiognomie der Riesenzellensarkome bei "Ostitis fibrosa" hervor. Nicht so unbestreitbar ist.es dagegen, dass ihre Besonderheit genügt, um die Annahme ihres entzündlichen Ursprungs zu rechtfertigen und festzustellen. Jedenfalls würde es sich dabei um eine durchaus seltsame Art von phlogistischen Produkten handeln, denn wenn auch stellenweise im Granulationsgewebe und in den spezifischen infektiösen Granulomen spärliche isolierte Riesenzellen $\mathrm{zu}$ finden sind, hat man doch nie Gelegenheit, 
solche typischen, bestimmten und nahezu autonomen riesenzellensarkomähnlichen Bildungen zu finden, wie bei der Ostitis fibrosa.

Und wenn man der Anschaung von Lubarsch beitreten wollte, welche übrigens sehr genial und anziehend erscheint, mïsste man noch erklären, warum solche Einschmelzungs- und Resorptionsbildungen nicht bei allen pathologischen Prozessen der Kuochen gefunden werden, bei welchen eine aktive lakunäre Zerstörung stattfindet, und besonders warum sie bei der jugendlichen umscbriebenen Varietät der v. Reckinghausenschen Knochenkrankheit so selten sind, wo doch die Knochenrarefaktion eine so ausgesprochene ist und es deshalb - wie es sonst die Häufigkeit der spontanen Frakturen beweist - nicht an stark eingeschmolzenen Knochenstellen fehlt.

Uebrigens ist wahrscheinlich die Anomalie im Verbalten der oftgenamnten Geschwülste eine geringere, als man auf den ersten Blick annehmen kö̀nte. Das wurde auch in der deutlichsten Weise von v. Haberer in der kritischen Uebersicht der genau untersuchten Fälle von diffuser Ostitis fibrosa bewiesen, welche er in seiner wertvollen, in dem Archiv für klinische Chirurgie erschienenen Arbeit ${ }^{1}$ ) gibt.

Auch aus unserer Tabelle geht hervor, dass ein mehr oder minder zahlreiches Vorkommen von Riesenzellensarkomen bei allen tödlich geendeten Fällen der verbreiteten v. Recklinghausenschen Knochenkrankheit zu beobachten ist, mit Ausnahme eines einzigen Falles (Fall $\mathrm{V}$ von v. Recklinghausen), bei welchem der Exitus durch eine von der Osteopathie unabhängige Krankheit herbeigeführt wurde. Nun erfolgte in der Mehrzahl der zugrunde gegangenen Fälle der Tod unter kachektischen Erscheinungen, wahrscheinlich als direkte Folge der Osteopathie, nach einer zwischen 5 Monaten und 11 Jahren schwankenden Verianfsdauer.

v. Haberer hebt noch das ganz verschiedene Verhalten der Sarkome und der Fibrome in bezug auf die Cystenbildung hervor, wie es aus Mönckebergs Beobachtung erbellt:

„Während.... in der Umgebung der Riesenzellensarkome und der aus ihneu entstandenen Cysten stets eine lebhafte lakunäre Knochenresorption zu beobachten ist, fanden sich an der Peripherie der fibrösen Herde und der Cystofibrome Vorgänge excessiven Knochenanbaues. Die lebhafte Knochenbildung bringt es mit sich, dass, wenn die Cystenbildung zum Stillstande gekommen ist, direkt ausserhalb der dünnen, fibrösen Innenwand ein dichtes Gefüge spongiöser Knochensubstanz zu sehen ist, welches wohl dem weiteren Wachstum Eiuhait getan hat. Im Gegensatz dazu haben die Riesenzellensarkome und die aus ihnen entstandenen Cysten infolge der in ihrer Umgebung stattgefundenen lakunären Knochen-

1) Zur Frage der Knochencysten. Arch. f. klin. Chir. Bd. 82 . S. 873. 
resorption eine viel grössere Wachstumsfähigkeit. Wir sehen sie bis an das Periost vordringen und dieses noch halbkugelig vorbuchten."

Die Inaktivität und die lokale Gutartigkeit der fibrös-ostitischen Riesenzellensarkome sind also stellenweise sehr relativ.

Wie man sieht, müsste man, auf Grund von Mönckebergs Beobachtung, viel mehr das Ausgeprägtwerden der Knochenresorption auf die Anwesenheit der Riesenzellensarkome; als die Entstehung dieser letzteren auf die osteoklastische Zerstörung bezjehen.

Weitere Beweise für die fortschreitende Natur dieser eigentümlichen Sarkomatosis und für die Neigung derselben zur Ausbreitung hat v. Haberer noch aus den radiographischen Untersuchungen gewonnen, welche er einige Jahre nach der ersten Untersuchung an seinen Kranken Gelegenheit hatte auszuführen, indem er das erhebliche Wachstum in loco der älteren Herde und das Erscheinen von neuen Herden in zahlreichen bis dabin gesunden Knochen beobachten konnte.

v. Haberer gibt wohl zu, dass, während das Vorkommen jüngerer Lokalisationen an eine richtige Metastase aus den primären Herden denken lässt, es doch eigentümlich erscheint, dass diese "die teilweise von einer unendlich dünnen Kortikalis gedeckt waren, trotz nachgewiesener teilweiser Vergrösserung diese dünne Decke nirgends durchbrochen hatten."

Ein solches Verhalten - fügt er hinzu - gehört doch, obwohl auch echt myelogene Riesenzellensarkome der Knochen zuweilen eine geringe Neigung zum Ùebergreifen auf die umgebenden Weichteile zeigen können, aussschliesslich den sarkomartigen Bildungen der Ostitis fibrosa. Handelt es sich um eine durchaus eigentümliche, klinisch anomale Art von Riesenzellensarkom, oder verlieren die myeloiden Sarkome, wegen ihrer Verbindung mit der Osteomalazie, ihre sonstigen Eigentümlichkeiten? Diese Frage lässt v. Haberer ungelöst.

Jedenfalls gewinnt er aus den obigen Betrachtungen die Ueberzeugung, solchen besonderen Produkten der Ostitis fibrosa den pathologischen Wert echter Sarkome zuerkennen zu dürfen, in Uebereinstimmung mit ihrem unzweifelhaft sarkomatösen morphologischen Aussehen.

v. Haberers Anschauungen muss man um so mehr als rationell begründet ansehen, als bisher kein sicheres Merkmal einer entzündlichen Entstehung dieser Tumoren angegeben wurde.

Dass die Multiplizität der Lokalisationen nicht immer eine primäre ist, sondern stellenweise das Aussehen einer wirklichen metastatischen Bildung anzunehmen scheint, dafür sprechen, ausser dem Fall von v. Haberer, diejenigen von Mönckeberg bzw. von Lissauer.

Im ersteren waren, mehrere Jahre bevor sich die Erscheinungen des diffusen Knochenleidens entwickelten, wiederholt Geschwülste an den Kiefern exstirpiert worden. Erst für die zuletzt, 6 Jahre vor dem Tode 
entfernte Geschwulst konnte die auf ein Osteosarkom lautende Diagnose festgestellt werden. Die Patientin starb im Alter von 55 Jahren. In den letzten 19 Lebensmonaten entstanden bei der sehr herabgekommenen Frau zahlreiche spontane Frakturen.

Anatomischer Skelettbefund: Multiple Cystenbildung; in der Umgebung der Cysten faserige Umwandlung des Markes, fibromartige und riesenzellensarkomähnliche Herde.

Bei Lissauers Fall ging dem Auftreten der Knochenverdickungen und der Spontanfrakturen an übrigen Skelettabschnitten ein Myeloidsarkom am Mittelgliede des rechten Zeigefingers voran. Leider wurden nach dem Tode nur wenige Knochenstücke untersucht; es ergab sich daraus nichts anderes, als ein hochgradiger Knochensehwund und die Umwandlung des Knochenmarkes in faseriges Gewebe.

Dieser Fall würde an und für sich nichts beweisen; wenn man ihn aber mit demjenigen von Mönckeberg in Beziehung setzt, trägt er mit demselben dazu bei, die Möglichkeit einer Beziehung zwischen den zuerst entstandenen isolierten Tumoren und der später entwickelten diffusen Osteopathie nachzuweisen.

Unsere Bemerkung, dass bei der jugendilichen umschriebenen Form der v. Recklinghausenschen Knochenkrankheit, und namentlich bei den dazugehörenden Beobachtungen, welche mit einem vollkommenen, oder mindestens genügend ausgedehnten mikroanatomischen Befunde versehen sind (Fälle von Küster, Bockenheimer, Tietze), echte Riesenzellensarkome in der Regel fehlen ${ }^{1}$, stimmt mit v. Haberers Auffassungen überein, und bestätigt sogar dieselben in indirekter Weise.

Wir müssen zwar zugeben, dass die Zahl der kompletten Beobachtungen, auf welche sich unsere Anschauung stützt, eine geringe ist. Ebenso ist die Einwendung zu erwarten, dass bei einigen jüngst veröffentlichten

1) Nur zwei Fälle von örtlicher Ostitis fibrosa mit Riesenzellensarkomen wurden uns bis jetzt zur Kenntnis gebracht. Der eine (Bötticher - Mönckeberg, 1905) betrifft eine multilokuläre Cyste am oberen Ende des rechten Oberarmes bei einer 25jährigen Frau; andere deutliche Herde waren nirgends radiographisch nachweisbar. In klinischer Hinsicht sprach das Aussehen der Neubildung für ein Osteosarkom; auf Grund solcher Diagnose wurde eine sehr breite Resektion des Knochens ausgeführt. Die histologische Untersuchung des entfernten Stückes ergab im wesentlichen: Umwandlung des Markgewebes in Fasermark; fibröse Knochenneubildung; riesenzellensarkomartige Herde.

Auch beim zweiten Falle (Pfeiffer, 1907, Cyste am unteren Femurende einer 20jährigen, wenige Monate nach der Operation an Lungentuberkulose gestorbenen Frau) lautet die mikroanatomische Diagnose: „entzündlich infiltriertes Riesenzellensarkom; typische Veränderungen der Ostitis fibrosa in den umgobenden Knochenteilen." 
Fällen von lokalisierter Ostitis fibrosa nicht die Möglichkeit a priori ausgeschlossen werden kann, dass sich die Krankheit mit der Zeit auf andere Skelettanteile verbreite, und die Einwendung, dass man bei anderen solcher Fälle nicht sagen kann, welches der weitere Verlauf der Krankheit gewesen wäre, wenn er nicht durch den Tod unterbrochen worden wäre (Fälle von Küster und von Bockenheimer) oder wenn nicht durch einen ziemlich radikalen operativen Eingriff der anfängliche Herd entfernt worden wäre (Fall von Tietze).

Andererseits liegt ein Fall von v. Recklinghausenscher Knochenkrankheit vor, den wir zu den typischen gerechnet haben, bei welchem keine Riesenzellensarkome, sondern nur die Cystofibrome gefunden wurden, und nichtsdestoweniger der Prozess auf einen grossen Teil des Skeletts verbreitet war: das ist der Fall $V$ von v. Recklinghausen. Hier kann nicht die Metastasenbildung zur Erklärung der Multiplizität der Lokalisationen herangezogen werden, sondern wir müssen annebmen, dass ein allgemeiner dystrophischer Zustand des ganzen Skeletts in allen Anteilen dieses letzteren Bedingungen geschaffen hatte, welche der Entstehung multipler, gleichartiger, aber voneinander unabhängiger Veränderungen günstig waren. Wenn man bedenkt, dass auch bei den Formen mit Entwick]ung von Riesenzellensarkomen dasselbe prädisponierende Moment (Osteomalazie) mitwirkt, begreift man, dass man allein auf den anscheinend metastatischen Charakter keine Schlussfolgerungen stützen kann.

Wir wollen jedoch der eben erwähnten Feststellung keine übermässige Bedeutung zuschreiben und nur bemerken, dass dieselbe ein neues Argument zugunsten der v. Habererschen Auffassung liefert, ein Argument, welches durch seine Assoziation mit anderen Zeichen und Gründen einen gewissen Wert annimmt.

Ich habe mich bisher damit beschäftigt, die Tatsache hervorzuheben, dass im jugendlichen Alter die tumorbildende Ostitis fibrosa am öftesten den Charakter eines örtlichen bzw. eines diffusen Vorganges aufweist. Dies glaube ich nun gewissermassen mit der habituellen Nichtteilnahme des riesenzellensarkomatösen Elementes in Zusammenhang bringen zu dürfen, welches bei dem anatomischen Bilde der diffusen Formen fast immer in den Vordergrund tritt.

Rebns und v. Haberers Beobachtungen scheinen beide klar dahinzuweisen, dass, wenn bei der jugendlicben Ostitis fibrosa die Riesenzellensarkome auftreten, die Osteopathie sich in demselben Masse verbreitet, wie bei der späteren Form und mit einer gewissen Rapidität fast das ganze Skelett befällt $\left.{ }^{1}\right)$.

1) Ausser den eben erwähnten Fällen lisgt uns noch einer pon multiplen, zerstreuten, im Laufe von vier Jahren bei oiner 24jährigen Frau entwickelten 
Die übrigen Abweichungen der jugendlichen Form vom typischen v. Recklinghausenschen Bilde, d. h. das vorzugsweise Befallensein der metaphysären Knochenabschnitte und das Vorbandensein von Knorpel inmitten des nengebildeten tumorartigen Gewebes, sind auch Erscheinungen von höchster Wichtigkeit, mit welchen wir uns weiter unten in bezug zu unseren Fällen beschäftigen werden müssen. Hier soll nur darauf hingewiesen werden, dass dieselben an die Möglichkeit eines Zusammenhanges der Erkrankung mit Störungen der enchondralen Verkuöcherung denken lassen. Mit dieser Auffassung verbinden sich verschiedene pathologische Deutungen des Prozesses, welche hier kurz erwälnt werden müssen.

Auf der Naturforscherversammlung 1904 stützte sich $\vee$. Mikulicz auf den damals erst zur Diskussion gebrachten Fall von v. Haberer, auf vier eigene ${ }^{1}$ ) und auf mehrere andere in der Literatur enthaltene ähnliche Fälle ${ }^{2}$ ), um die Definition eines besonderen Krankheitsbildes festzustellen, dessen vermutliches pathologisches Wesen mit der von ihm vorgeschlagenen Bezeichnung: Osteodystrophia cystica (juvenilis) angedeutet wird. In seinen Hauptlinien entspricht das in Betracht gezogene Bild genau demjenigen der jugendlichen umschriebenen tumorbildenden Ostitis fibrosa: "Es ist eine Krankheit der Wachstumsperiode... betrifft vorwiegend die langen Extremitätenknochen und zwar diejenigen Abschnitte, welche der Zone des stärksten Wachstums entsprechen ... In den meisten Fallen spielt ein initiales Trauma eine Rolle... Es ist ein durchaus gutartiger Prozess ... Die Cystenbildung kommt meist isoliert, in selteneren

Knochencysten vor, deren Abstanmung von einer Ostitis fibrosa doch sehr zweifelbaft bleibt; der Fall wurde von Heinecke (Beitr. z. klin. Chirurgie, Bd. 40, H. 2) geschildert, und die Erkrankung, um welche es sich dabei handelt, als eine der v. Reckling hau sen schen Ostitis deformans nảhestehende allgemeine Skelettkrankheit aufgefasst, obwohl die charalteristischen Knochenverkrümmungen allerdings dabei fehlten. Nur ein excidiertes Stückchen Cystenwand wurde mikroskopisch untersucht; in demselben waren weder Riesenzellensarkome noch irgend andere tumorartigen Bildungen, sondern nichts anderes als faseriges Bindegewebe und Knochenbälkchen nachzuweisen.

1) Bei v. Mikuliczs Fällen, welche alle jüngere Leute betreffen, handelt es sich zweimal um isolierte Cysten am oberen Humerusende und einmal um eine Cystenbildung im obersten Femurende, mit einer gleichzeitigen, weniger ausgeprägten Veränderung im Unterschenkel. Beim vierten Falle bestand die Krankheit in einer cystischen Entartung der beiden oberen Femurenden und in ähnlichen, aber leichteren Veränderungen in mebreren anderen Knochen. Es handelte sich also um drei lokalisierte und eine verbreitete Form.

2) Letztere wurden von Tietze in seiner Arbeit "Ueber Knochencysten" (Beitr. z. klin. Chirurgie, Bd. 52, H. 2) gesammelt und ausführlich wiedergegeben. 
lällen multipel, in voneinander entfernten Skeletteilen vor. Neben vollkommener Cystenbildung findet man an anderen Stellen eine Art Vorstadium oder ein unentwickeltes Stadium, in welchem das Knochengewebe zum Teil durch Bindegewebe (osteoides Gewebe?) mit zahlreichen Riesenzellen substituiert ist" ${ }^{\text {) }}$.

Während v. Haberer seine Beobachtung unter dem Titel „Multiple Knochentumoren" vorstelite, und auch frühere Beobachter (Körte, Schlange, König) bei lokalen cystenbildenden Knochenerkrankungen das Aussehen und das Verhalten eigentlicher Geschwülste wahrgenommen zu haben dachten, und dementsprechend dieselben als cystische Enchondrofibrome betrachteten, war v. Mikulicz zu der Ueberzengung gelangt, dass solche Erklärung für dieses sonderbare Leiden nicht zutreffend sei. Es würde sich vielmehr um eine „eigenartige Störung in der Entwicklung des im Wachstum befindlichen Knochens" handeln.

Leider konnte v. Mikulicz diese Auffassung, welche in ihrem arsprünglichen Entwurfe etwas unbestimmt erscheint, nicht weiter ausarbeiten bzw. entwickeln und genauer bestimmen. Dieselbe wurde dann von Glimm und Tietze verteidigt und illustriert, welche, indem sie das pathologischanatomische Gleichwertigsein der zur Entwicklung der sogenannten echten Knochencysten führenden Vorgänge und derjenigen der tumorbildenden Ostitis fibrosa anerkennen, die erstereu als zu einer Krankheit sui generis gehörend betrachten, für welche ihnen die Bezeichnung: Osteodystrophia cystica angepasst erschien.

v. Mikuliczs Anschauungen folgend, stimmen Glimm²) und Tietze darin überein, dass sie dem Prozesse jede neoplastische Deutung (in engerem Sinne) absprechen, und ihn vielmehr für eine eigentümliche Entzündung halten. Es wäre, nach Tietzes Ansicht, eine besondere entzündliche Wucherung, welche bei meistens jugendlichen, in ihren Entwicklungs- und Wachstumsfähigkeiten sonst veränderten Knochen zustande kommt. "Aus irgend einem uns zunächst unbekannten Grunde kommt es zu einer krankhaften Affektion dieses Knochens, einer Störung seines Gefüges. Der normal veranlagte Knochen würde seine Struktur allmählig wieder herstellen. Dazu ist num aber - und das ist seine Krankheit - der cystenbildende Knochen nicht imstande, sondern er bringt es nur zu Ansätzen, zu einer gewissen Nachahmung der normalen Konfiguration" ${ }^{3}$ ).

Schon 1903 suchte Beck ${ }^{4}$ ) das Wesen des cystenbildenden Vorganges

1) v. Mikulicz, Zentralbl. f. Chirurgie. 1904. No. 46. S. 1323.

2) Zeitschr. f. Chir. Bd. 80. S. 476 .

3) Tietze, a. a. 0. S. 552.

4) Beck, Ueber echte Cysten der langen Röhrenknochen. Archiv f. klin. Chir. Bd. LXX. 1903. 
in einer Rachitis und der Osteomalazie sehr nahe stehenden entzündlichen Atrophie; diese sollte als Reaktionserscheinung auf eine abnorme Wucherung von metaphysären. Knorpelresten folgen, bedingt durch irgend einen besonderen Reiz, meistens ein Trauma.

Nach dem Berichte meiner eigenen Beobachtungen werde ich mich mit der Bedeutung dieser Erklärungsversuche und mit der Frage nach der Pathogenese der Erkrankung beschäftigen müssen. Was nun ihren Platz in dem Kapitel der Knochenkrankheiten, also ihre doktrinale Klassifikation anbetrifft, mus mản annehmen, dass diesbezüglich die meisten Autoren der Cystenbildung eine zu grosse Wichtigkeit bzw. Bedeutung zugeschrieben haben. Nach meiner Ansicht - und darin stimme ich vollständig mit Gaugele ${ }^{1}$ ) überein - müsste man den Ausdruck echte Knochencysten und jede andere gleiche Bezeichnung absolut fallen lassen.

$\mathrm{Ob}$ man nämlich die Knochencysten als das direkte Produkt eines Schwundes des Knochengewebes, oder als das Endresultat irgend eines Umbildungsvorganges von krankhaften Gefügen ansieht, stellen dieselben doch immer eine sekundäre Erscheinung dar, und können nicht eine besondere selbständige krankhafte Entität charakterisieren.

Erstens ist es wohl festgestellt, dass die mehrfach bestrittene Virchowsche Lehre - Entstehung der gutartigen Knochencysten aus erweichten Enchondromen - für manche besondere Formen noch gelten kann. Nach Tietze würde sie namentlich nicht nur für den typischen Fall von Virchow, sondern auch für denjenigen von Helbing und für einige unter den Boströmschen ${ }^{2}$ ) Fällen am besten passen. Lexer ${ }^{3}$ ) verteidigt dieselbe in sehr ïberzeugender Weise und zwar auf Grund einer eigenen Beobachtung. Neben diesen mit grösserer Wahrscheinlichkeit als echt neoplastischen Ursprungs zu haltenden Formen, und abgesehen von den rein traumatischen Cysten, deren Vorkommen übrigens von manchen Autoren - z. B. von Lexer - mit Recht angezweifelt wird, findet man die Cysten bei der jugendlichen umschriebenen v. Recklinghausenschen Knochenkrankheit oder nach Belieben, die osteodystrophen Cysten v. Mikuliczs.

Wir glauben als letzteren nahestehend diejenigen Cysten betrachten zu können, welche man bei der typischen, bei erwachsenen und älteren Leuten vorkommenden Form der tumorbildenden Ostitis fibrosa beobachtet, obwohl sie, wie Tietze mit Recht bemerkt, in diesem Krankheitsbilde eine viel geringere Bedeutung annehmen, als bei den jugendlichen Formen, wo

1) G gele, a. a. 0 .

2) Boström, angef. von Tietze.

3) Lexer, Ueber die nicht parasitären Cysten der langen Röhrenknochen. Archiv f. klin. Chir. Bd. 81. H. 2. S. 363. 
sie es oft vollständig beherrschen. Endlich wurden ja Cysten bei echter Osteomalazie (v. Recklinghausen, Rindfleisch) bei Osteoarthritis deformans (Ziegler), bei örtlichen infektiösen Knochenerkrankungen [v. Braun] $]^{1}$ - abgesehen von der bekannten Periostitis albuminosa beobachtet. Dennoch genügt die blosse Ausschliessung aus diesem Gebiete der parasitären (Echinokokken) und der aus echt bösartigen Tumoren entstandenen Cysten überhaupt nicht, um dem Begriffe "gutartige bzw. echte Knochencysten" jene einheitliche Auffassung zu verleihen, welche für die Bestimmung eines besonderen Krankheitsbildes erforderlich ist.

Wenn wir andererseits die Cystenbildung als einen wesentlichen Charakter der Osteopathie betrachten, so müsste man aus ihrem Bereich eine gewisse Anzahl von übrigens genau damit übereinstimmenden Fällen nur darum ausschliessen, weil keine Cysten bei ihnen nachweisbar waren.

Nun ist es leicht begreiflich, dass gerade diese Fälle den krankhaften Prozess in einer Periode seiner Evolution (unentwickeltes Stadinm, v. Mikuliczs Vorstadium) darstellen, welche sich der pathologischen Deutung günstiger erweisen als das cystische Stadium. Es liegen uns bei jenen Fällen die ursprünglichen Veränderungen, d. h. die Prozesse vor, welche der Cystenbildung vorangehen und die Cysten sozusagen vorbereiten. Sie bestehen im Grunde genommen aus zwei Elementen: Resorption des Knochengewebes, Fibrosis des Knochenmarkes mit osteoider Neoproduktion, Entwicklung von geschwulstartigen Neubildungen; letztere zwei Elemente sind oft, wie wir sehen werden, schwer unter sich differenzierbar. Dieselben Elemente erkennt man in den vorgeschrittenen Fällen neben den Cysten; nur können sich die primären Veränderungen, wenn die Cysteı eine sehr grosse Entwicklung genommen haben, auf wenige Spuren reduzieren oder gar vollständig verschwinden. Jedenfalls muss man auf Grund der genannten Elemente nach der Natur des Prozesses forschen und denselben klassifizieren.

Da nun der oben kurz dargestellte anatomisch-pathologische Komplex der gleiche wie bei der v. Recklinghausenschen Knochenkrankheit ist, liegt nach meiner Ansicht kein Grund vor, um diese Formen auszusondern, welche man $\mathrm{ja}$ als jugendliche umschriebene Varietäten der v. Recklinghausenschen Erkrankung ansprechen kann, obne sie getrennt unter irgend einer anderen Benennung zu sammeln.

Fall I. Ein 17jähriges, gesundes, auf dem Lande lebendes Mädchen wurde am 9. Oktober 1906 in unserem Institute aufgenommen. Erblich nicht belastet; nichts Bemerkenswertes in der Anamnese, wenn man von einem 3 Jahre vorher durchgemachten Typhus absieht; keine Zeichen von Rachitis. Im Frühjahr 1904 war

1) v. Braun, Ueber Cysten in den langen Röhrenknochen. Beitr, zur klin. Chir. Bd. 52. H. 2. 
Patientin, welche bis dahin keine Funktionsstörungen der unteren Extremitäten und keine Anomalien derselben aufgewiesen hatte, von einem Maulbeerbaum auf den Boden und dabei auf die linke Hüfte gefallen. Nach den Angaben der Patientin, welche sich sebr genau des Vorkommnisses und seiner Folgen erinnert, kann man mit ziemlicher Sicherheit ausschliessen, dass eine Fraktur des Femur stattgefunden babe; nach 6 Tagen Bettruhe konnte Patientin mit Hilfe eines Stockes gehen und einige Tage später auch ohne denselben. Zu dieser Zeit empfand sie nur eine leichte'Schmerzhaftigkeit an der Stelle des Traumas, d. h. im oberen Drittel der Femurdiaphyse. Nach 2 oder 3 Monaten merkte sie, dass sich eine Verdickung. und ein kantiger Vorsprung am Knochen gebildet hatten, welche nach und nach zunahmen, während das ganze Glied sich allmählich verkürzte und der Gang ein hinkender wurde.

Als Patientin im Institut aufgenommen wurde, wies die linke untere Extremität eine Verkürzung von etwa $8 \mathrm{~cm}$ auf, welche hauptsächlich auf eine ausgesprochene Krümmung der Femurdiaphyse nach vorn und nach aussen zurückzuführen war, deren Gipfel der unteren Grenze des oberen Femurviertels entsprach. Dieser Gipfel bildete eine oberflächliche Erhabenheit ungefähr $10 \mathrm{~cm}$ unter dem Troch. major und entsprach dem hervorspringendsten Punkte der Konvexiät, indem letztere stark übertrieben erschien (Fig. 1).

Der Schenkelknochen fand sich bei der Palpation entsprechend derDeformation diffus vergrössert, nicht schmerzhaft; die Resistenz erschien auch in allen betastbaren Punkten normal. Es wurde auch eine mässige Hypotrophie der Muskeln des Gliedes, ein kompensatorischer Equinismus des entsprechenden Fusses, habituelle Flexion des andern Knies und statische Obliquität des Beckens wahrgenommen.

Die Radiographie (Tafel XVII, Fig. 2) zeigte, dass die Deformität sich auf den voluminösen und in starker Varusstellung befindlichen Femurhals erstreckte; dabei wurden Zonen von ausgesprochener Knochenrarefaktion in der Höhe der Krümmung des Knochens und in den umliegenden Diaphysenteilen beobachtet. Dagegen fand man im Radiogramm entsprechend den unteren Umrissen des Halses und dem medialen Teil der Diaphyse einen sehr dunklen Schatten, welcher auf eine grössere Kompaktheit der Corticalis hinzuweisen scheint, bedingt entweder durch Nachbarschaftsreaktionsvorgänge, oder durch die abnorme Stimulation der Belastung, welche bei den neuen mit der Deformität zusammenhängenden statischen Verhältnissen auf den medialen Teil des Knochens ausgeübt wird.

Aehnliche Veränderungen des Röntgenbildes werden in den wenigen Fällen von Pagetscher Ostitis deformans und von Ostitis fibrosa beobachtet, welche wir in den vorliegenden Atlanten finden konnten.

Unser Befund erlaubte uns jedoch nicht, einen zentralen Tumor des höheren Femuranteiles auszuschliessen; da aber die ziemlich langsame Evolution der Krankheit nicht ein myelogenes Sarkom vermuten liess, wurde ein palliativer Eingriff $\mathrm{zu}$ orthomorphischem Zwecke vorgeschlagen bzw. vorgenommen. Die Operation wurde yon Prof. Dr. Galeazzi am 27. Oktober 1906 ausgeführt und bestand in einer keilförmigen Osteotomie (S. $407 \alpha$ und $\beta$ ) am Gipfel der Knochenkrümmung und in der darauffolgenden Immobilisierung des Gliedes in korrigierter Position und Richtụng. Nach einem Monat war der Kallus noch nichț vollständig konsolidiert; 
Figur 1.

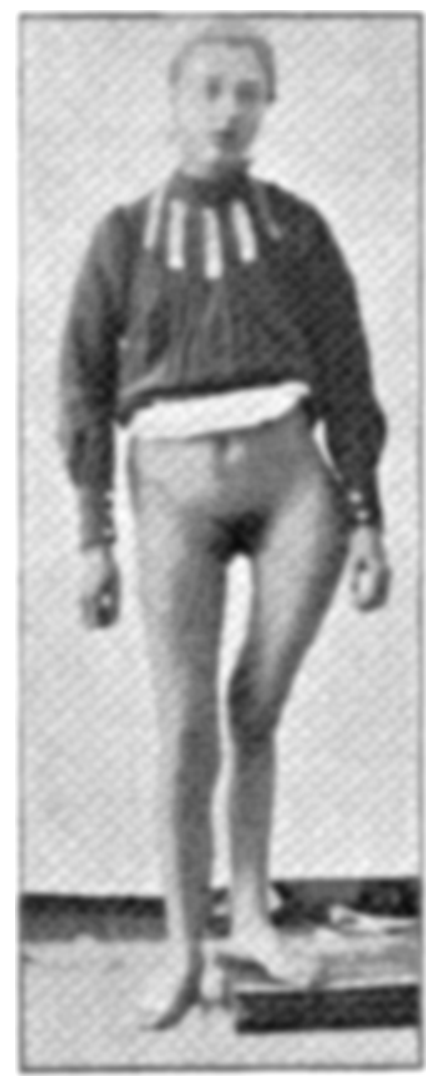

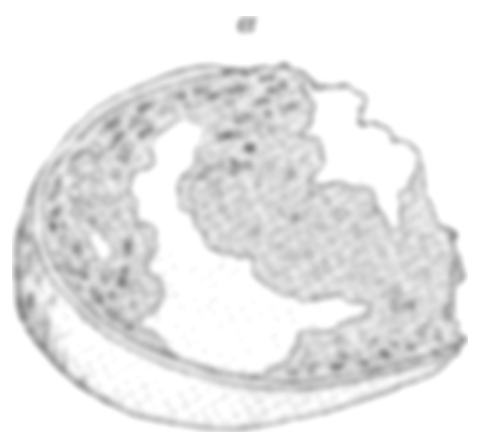

Osteotomischer Keil (von unten gesehen).

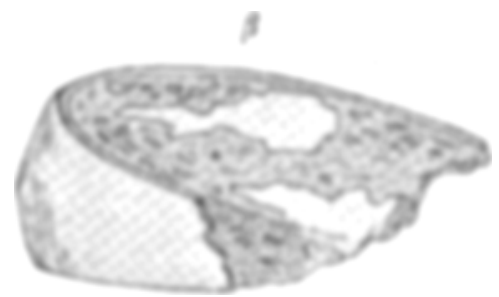

Osteotomischer Keil (von oben gesehen). 
es wurde wieder ein ambulatorischer Gipsverband angelegt und 50 Tage lang in locs gelassen, wonach die Konsolidation als eine perfekte erkannt wurde. Das Resultat war sowohl vom funktionellen wie rom kosmetischen Standpunkt aus ein ausgezeichnetes. Die Verkürzung des Gliedes war auf $3 \mathrm{~cm}$ reduziert (s. Tafel XVII, Fig. 3, und Textligur 4).

Bei der Operation leistete das Knochengewebe dem Schnitte einen geringen Widerstand, mit Ausnahme der medialen Wand des Diaphysenzylinders. Die

\section{Figur 4.}

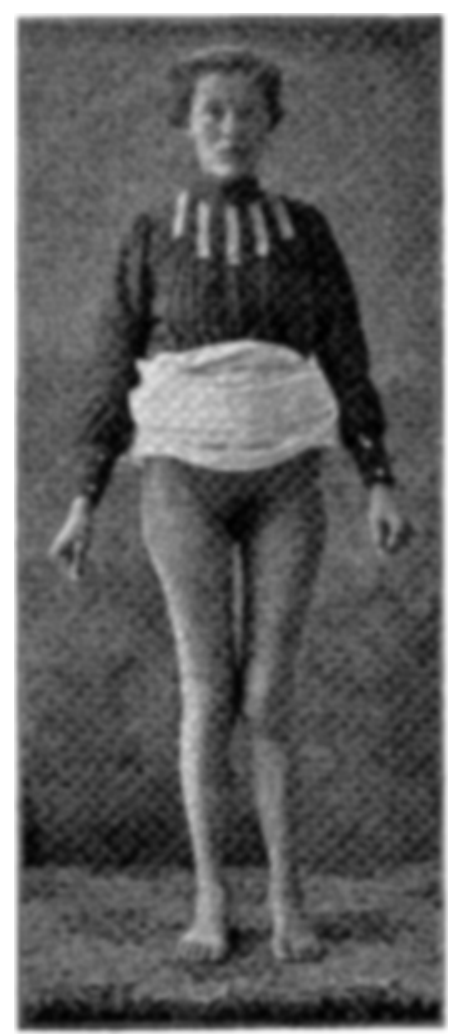

Corticalis war in allen übrigen Teilen der Peripherie sehr dünn, sie erreichte nirgends eine Dicke ron $1 \mathrm{~mm}$. In dem bei der Operation angegriffenen Segment fehlte jede Spur von Marlklkanal; die zwei Schnittflächen ersohienen in ihrer ganzen Ausdehnưung, bis zur Spitze des Keiles, durch spongiöses Knochengewebe eingenommen, spongiös rarefiziert an der Peripherie, dioht und homogen im Zentrum. Anf dem Grunde der Spongiosa waren zwei breite, deutlich umschriebene, unregelmässige Zonen eines weissen, perlmutterartigen Gewebes von knorpeliger 
Konsistenz sichtbar. Kleine Inseln eines gleichen Gewebes sind hier und da, be* sonders in dem antero-externen Teil der Sohnitte, zerstrent.

Die histologisehe Untersuchang (Fig. 5) ergab, dass die Zonen, welche bereits makroskopisch ron dem umgebenden Knochen rerschieden erschienen, grösstenteils aus fibrösem, an spindelförmigen Zellelementen sehr reichem Gewebe bestanden, wolches hier und da von osteoiden Bälkchen durchsetzt war.

In einigen Zonen findet man dagegen sternförmige, weniger dichte, bindegewebige Elemente, versehen mit Ausläufern, die sich durchkreuzen und eine Art

\section{Figur 5.}

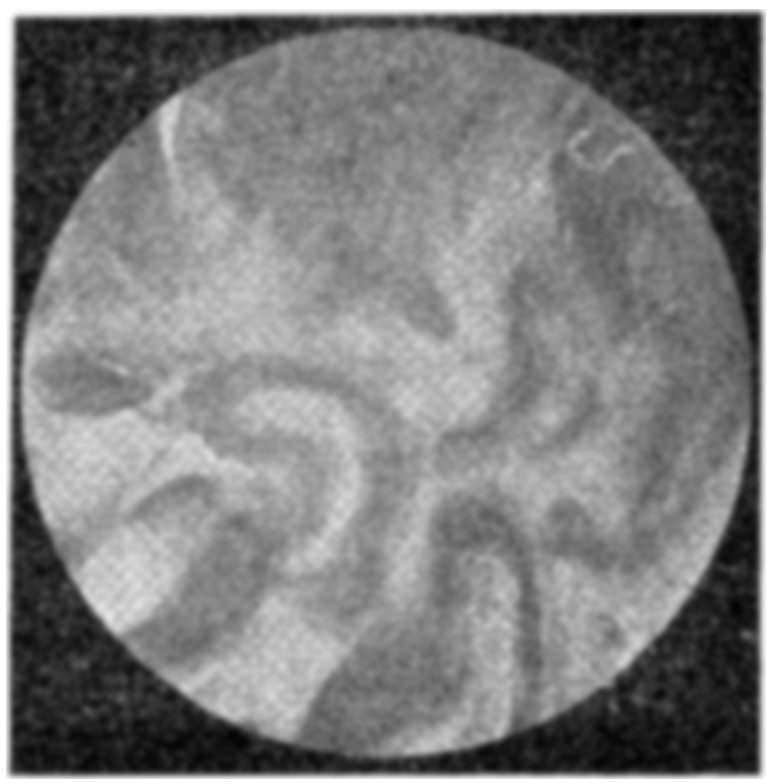

Markfibrose. Osteoklastischer Knochenschwund. Osteoide Bälkchen.

Ketz bilden, äbnlich wie man es bei den Knochenzellen und den Zellelementen der Hyxome findet. In überwiegendem Masse findet man jedoch die echtfibrösen Zonen.

Man erkennt ausserdem im Gewebe einige kleine Flächen ron Faserknorpel, deren Natur aus der intensiveren Färbung mit Hämatoxylin und mit Toluidinblau - mit letzterem ist auch die rötlich-violette Reaktion der mukösen Substanz zu beobachten - und aus der Tatsache crhellt, dass seine zellulären Elemento voluminös und deutlich gekapselt sind. Einzelne ähnliche gekapselte Zellen findet man im Fasergewebe und in den osteoiden Bälkchen verstreat.

An den Stellen, wo sich die fibröse Neubildung bis unter die Korticalis ausdehnt, finden wir eine Uebergangszone, charakterisiert durch die Anwesenheit von zahlreichen unregelmässigen Schollen aus Knochensubstanz, welche in Form von

Zeitschrift für Krobsforschung. 7. Bd, 2. Feft。 
Inseln von der bindegewebigen Zone umgeben und darin enthalten sind; in ihren Einbuchtungen enthalten diese Bälkchenreste zahlreiche Osteoklasten; die spindelförmigen Elemente des Bindegewebes sammeln sich um die Knocheninseln herum diohter als anderweitig an, während hier die fibrilläre, interzelluläre Substanz sehr wenig vorhanden ist. In der subkortikalenZone n̂ndet man auch Ansammlungen von Blutpigment und sehr wenige Riesenzellen, fast immer in der Nähe der zerfallenen und erodierten Knochenbälkchen. Die Korticalis zeigt keine kompalte Strultur mehr, sondern besteht aus einem unregelmässigen Netzwerk von ziemlich dicken Bälkchen, welche enge medulläre Räume umschreiben, aber auch selbst von alctiver, laknnärer Resorption befallen sind. Die kortikalen medullären Räume enthalten fast ohne Ausnahme ein faseriges, nioht sebr zellreiches Mark und keine Spuren von Fett- oder Lymphmark.

Der mit der zentralen Spongiosa, welche dieselben Veränderungen wie die kortikale aufweist, benachbarte Teil der Nenbildung enthält zablreiche dünne Bälkchen mit meist regelmässigen und nicht von Osteoklasten befallenen Rändern. Diesc Bälkchen, welche lieine deutliche lamelläre Struktur erkennen lassen und sich mit Karmin und mit Eosin gut färben, besteben böchstwahrscheinlich aus jungem osteoiden Gewebe.

Fall Il. Am 29. Juli 1907 wird im Institut ein 12jähriger Knabe, Bauernsohn, ziemlich kräftig, aufgenommen, bei welchem sich seit ungefähr einem .Tahre eine geringe Deformität des rechten Schenkelbeins mit Verkürzung des Gliedes und hinkendem Gang allmählich entwickelt hatte.

In der Anamnese ist betrefis Vererbung niohts zu finden, was mit der gegenwärtigen Osteopathie in Zusammenhang gebrachtwerden Könnte. Beim Patienten hatte die Zahnung zu richtiger Zeit stattgefunden, auch hatte er zur richtigen Zeit gehen gelernt. Im Alter von 4 Jahren hatte Patient eine Augenkrankheit gehabt, über welche die Angehörigen nichts Näheres angeben können; dieselbe war jedoch vollständig geheilt, ohne Spuren zu hinterlassen. Im März 1905 fiel Patient, indem er von einer mit Hen beladenen Karre horuntersprang, auf den Boden und stiess sich dabei die rechte Füfte. Er empfand dabei keinen grossen Schmerz, ronnte gleich aufstehen und ohne fremde Hilfe nach Hause gehen. Er war danach nicht bettlägerig. Es scheint sich in den nächsten lagen eine vorübergehende Ansehwellung der Leistendrüsen gebildet zu haben, während die ursprüngliche geringe Schmerzhaftigkeit der Hüfte einige Tage fortbestand.

Im I Jaufe einiger Tage verschwanden alle Beschwerden. Nach 6 Monaten entstanden dumpfe Schmerzen in der rechten Hüfte, welche besonders stark wurden, wenn Patient das Glicd dadurch anstrengte, dass er lange stand oder ging. Diese Beschwerden danerten oinige Wochen, worauf sie abnahmen und an Ende im Ruhezustand vollständig verschwanden.

Nun hat Patient seit ungefähr einem Jabr eine geringe Verkürzung des rechten Unterschenkels und ein anormales Vorspringen des Schenkelbeins in seinem oberen reile wabrgenommen.

Bex der objektiven Untersuchung des Patienten fand man geringe, jedoch deutliche Spuren yon rorausgegangener Rachitis. Hypsicephalie, unregelmässige Zähne, chondrokostaler Rosenkranz, Vergrösserung der Epiphysen der langen Knochen. Die bemerkenswerteste Skelettveränderung sass jedoch im liruralen 
Segment des rechten Unterschenkels (Fig.6). Dieses zeigte nämlich eine Verlürzung von $2,5 \mathrm{~cm}$, die entsprechende Muskulatur war, besonders am Schenkel, schmächtig und schlaff. Von der Seite gesehen, zeigte der Schenkel eine abnorme Konvexität in der subtrochanterischen Gegend; der Trochanter major war etwas nach hinten verlagert und stand etwa $1 / 2 \mathrm{~cm}$ höher als derjenige der linken Seite. Direlit unter dem Fortsatz ersehien die Femurdiapbyse bei der Palpation vergrössert und machte

Figur 6.

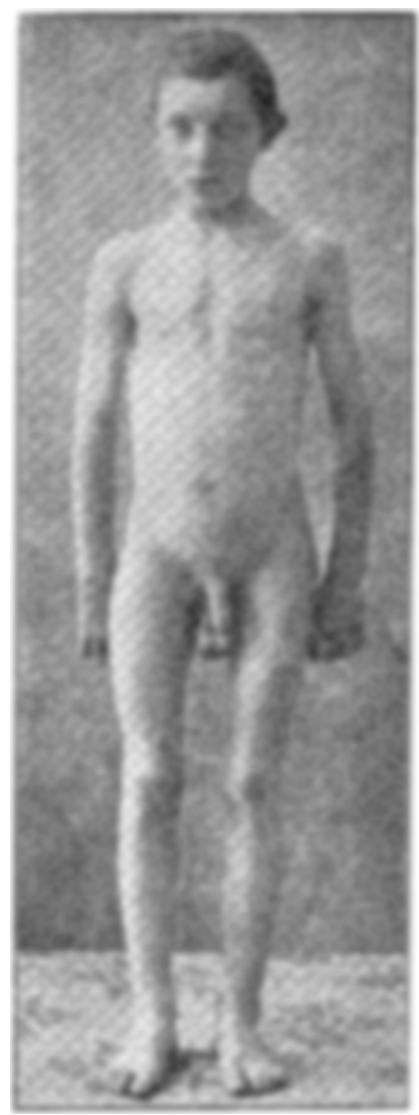

dabei sozusagen den Eindrnck eines voluminösen Frakturkallus. Die Vergrösserung nahm allmählich von oben nach unten spindelförmig ab. Nur auf der seitlich hinteren Fläche des Knochens hört sie, drei Querfinger unter dem Fortsatz, plötzlich auf, so dass eine $\Delta$ rt Stufe gebildet wird. Der übrige 'Teil des Schenkelbeins, welcher die drei distalen Viertel der Diaphyse umfasste, erschien von normalem Durchmesser; er folgte nicht dem vergrösserton Segmont in gleicher Richtung, 
sondern bildete mit demselben einen sehr stumpfen Winkel mit postero-lateraler Richtung; der obere Toil des Femurs erschien deslalb etwas vom Becken abduziert, der untere leicht flektiert. Die Bewegungen des Hüftgelenks waren keineswegs beschränkt bzw. gehemmt. Beim Stehen des Patienten beobachtete man eine Inklination des Beckens nach rechts, statische Lendenhyperlordose und -Skoliose, geringe äussere Rotation des Gliedes.

Bei der allgemeinen physikalischen Untersuchung wurden keine weiteren pathologischen Erscheinungen wahrgenommen.

Die Radiographie (Tafel XVII, Fig. 7) zeigt eine Verdickung des oberen Femurendes, welche jedoch den Femurkopf nicht betrifft und sich von der subkapitalen Gegend des Femurhalses bis zur unteren Grenze des oberen Viertels der Diaphyse erstreckt. Der Knochensohatten, welcher am oberen Rande des Halses und an der medialen Seite des erkrankten Diaphysensegments dicht und homogen ist, zeigt in den übrigen T'eilen der kranken Zone eine ausgesprochene Rarefaltion, welche am Halse und in der Trochantergegend in Maschenform erscheint. Im radiographischen Bilde erscheint das untere Profll des Femurhalses sehr unklar. Die Rarefaltion wird nach der Diaphyse hin einförmiger, letztere ist auf einer kurzen Strecke auch befallen und dadurch entsteht eine grosse Lücke im Schatten. Aus dieser Lücke streckt sich seitlich und nach oben ein Ausläufer vor, welcher der äusseren Korticalis des Femur folgt und den horrorspringendsten Punkt derselben unter dem Trochanter major erreicht.

So homogen anch die luioke ist, erkennt man in derselben unregelmässig rerteilte, scheidewandartige, dunklere Streifen.

Auch in diesem Fall wurde ein operativer Eingriff vorgenonmen (Professor Galeazzi) und am 8. August 1907 eine subtrochanterische, Keilförmige Osteotomie ausgefübrt. Der abgetrennte Keil, an der Basis $15-18 \mathrm{~mm}$ dick, reigte eine dünne und deformierbare Corticalis, and bestand grösstenteils aus dichtem, spongiösem Gerrebe. Nach dem Gipfel des Keiles fand man jedoch mehrere rund. liche, erbsengrosse Iinoten, von denen einige zu grösseren Nassen zusammenschmolzen und welche aus einem weissen, perlmutterähnlichen, genau an hyalinen Knorpel erinnernden Gewebe bestanden. Diese Knoten schienen mit der Spongiosa zusammenzuhängen; bei den Versuchen, sie zu cnukleieren, wurden kleine Knochenfragmente mitgerissen.

Bevor man die Sektion des Knochens durch Eraktur der medialen Korticalis vermittels gewaltsamer Akduktion des Gliedes vollendete, wurden aus der dureh Entfernung des Knochenkeiles erzeugten und bis etwa zur Mitte der Diaphysendicke sich erstreckenden Lücke mit einem scharfrandigen Löffel mehreve ziemlich voluminöse Fragmente eines knorpelartigen Gewebes entfernt.

Danach wurde das Glied in mittelmässiger Abduktion vermittels eines Gipsm verbandes immobilisiert. Eine perfelte Konsolidation des Kallus wurde nach 2 Monaten wahrgenommen. Die zurückgebliebene Verkürzung des Gliedes betrug $1 \mathrm{~cm}$, die Stellang and Funktionsfahigleit des Gliedes war ziemlich befriedigend.

Zur histologischen Untersuchung wurden sowohl die mil dem scharfen Löffel gewonnenen Fragmente wie der osteotomische Keil verwendet. Die ersten er* schienen grösstenteils aus Knorpel gebildet. Letzterer zeigt an einigen Stellen eine ziemlich regelmässige säulenförmige Anordnung seiner Elemente, wie es bei 
den interepiphysären Knorpeln der Fall ist, und eine hyaline, dentlich sichtbare Grundsubstanz; an anderen Stollen besteht er aus Ansammlungen von grossen vesikulären Kapseln, welche mebrere Kerne (bis zu 10) enthalten. Die mehr oder minder grossen Massen bzw. Ansammlungen sind in den Maschen eines spärlichen faserigen Stromas enthalten (Fig. 8).

An den Schnitten des Keiles interessieren zwei Knorpelknoten an ihrer Spitze; dieselben bieten die gleichen Charaktere dar, welche an den Fragmenten wahrgenommen wurden; man kann ausserdem deutliche Veränderungen des Knochengewebes erkennen. Die Korticalis ist im äussersten Grade verdünnt und auf wenige hier und da unterbrochene Lamellen des äusseren allgemeinen Systems reduziert;

Figur 8 .

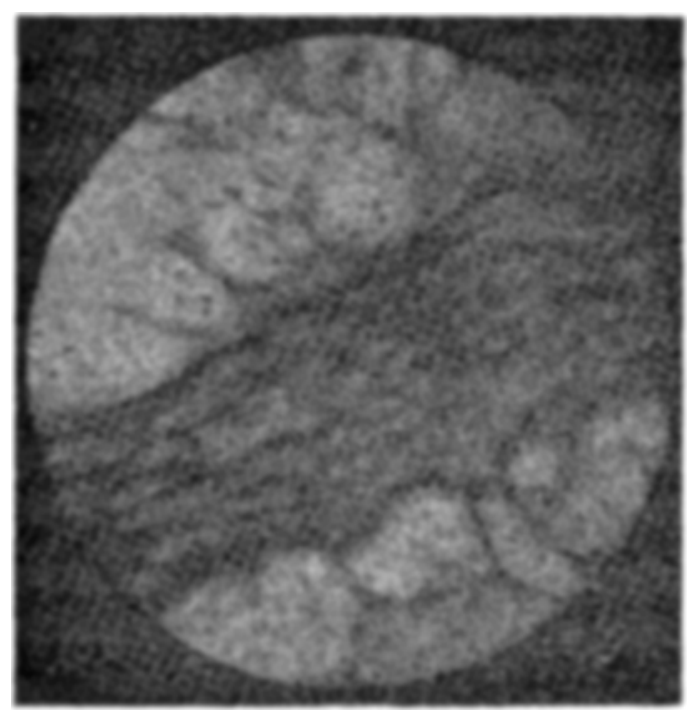

es fehlt somit eine wirkliche komparte Schicht; man kann das ganze Stück als aus Spongiosa bestehend betrachten. Diese weist breite, durch fünne Bälkohen begrenzte Markräume auf; die subliortikalen Räume enthalten noch Fettmark, einige adipös-lymphatisches Mark; alle übrigen sind nit einem fibrösen, an spindelförmigen, zellulären Elementen reichen Bindegewebe gefüllt. In einigen peripheren Markräumen lann man den Beginn der Fibrosis beobachten: während der grösste T'eil der Lücke durch normales Mark besetzt ist, sieht man an einer Ecke, meist um einen Gefässsohnitt herum, eine Zone fibrillären Gewebes, welche sich mit den Maschen des normalen Markes selbst fortzusetzen scheint (Fig. 9).

Die Erscheinungen der lakunären Resorption sind nicht sehr ausgesprochen, wenn man von der nächsten Umgebung der Knorpelknoten absieht; in dem übrigen Teil beobachtet man überall in vorwiegendem Masse die Halisteresis (Verdünnung 
der Bälkchen mit Vergrösserung und relativem anscheinendem Reichtum an Knochenlzörperchen). Es wird gleichzeitig eine aktive Apposition von osteoider kalkloser Substanz beobachtet. Vollständig osteoide Bälkchen sind selten; die Apposition geschieht meistens auf den alten Bälkchen in Form von mit Nentralkarmin sebr gut färbbaren Schichten, während der axiale kalkhaltige Teil des Bällkchens eine grössere Affinität für das Hämatoxylin zeigt. Diese Bällichen wurden durch Safranin nur im axialen Teil gefärbt; in allen Präparaten zeigen sie einen Suurn aus liontinuierlichen Osteoblastenreihen.

\section{Figur 9.}

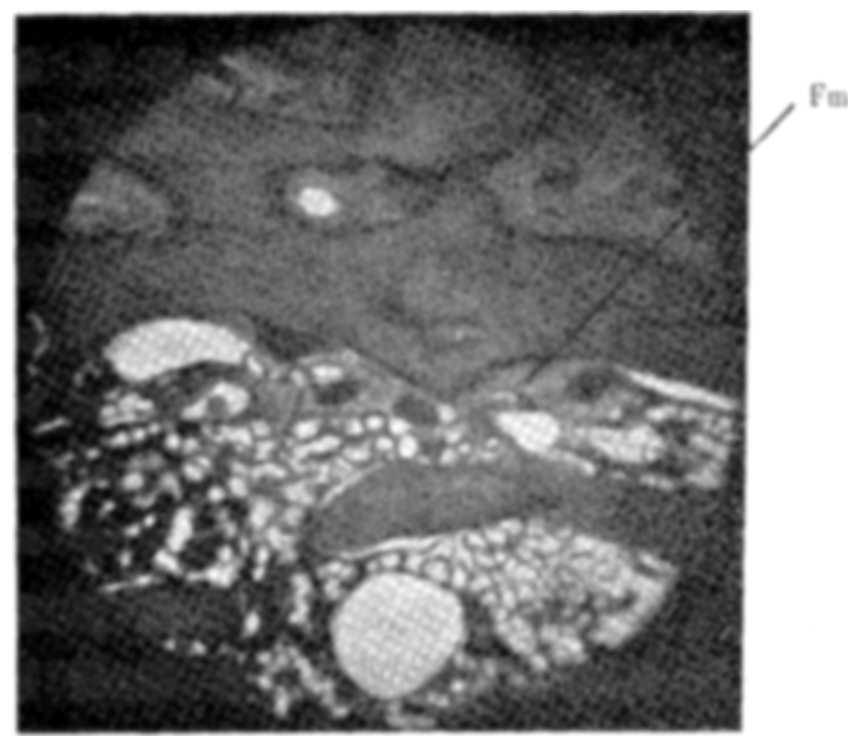

Beginnende Markfibrosis in den subkortikalen Markräumen. Fm Fasermark.

An der Peripherie der Knorpelzonen, welche sich rom Fasermark ganz unabhängig halten, beobachtet man einige kleine Hämorrhagien in Form ron Blutpigmentansammlungen.

In keinem Teile der Präparate findet man Riesenzellen, welche wegen ihrer unmittelbaren Beziehungen zu den arrodierten Knochenbälkchen nicht als in Alitivität sich befindende Osteoblasten betrachtet werden können. Uebrigens ist dieser Befund, wie bereits gesagt, auf wenige Pankte beschränlit.

Die zwei oben berichteten Fälle wurden von mir bei dem IV. Kongress der Societí ortopedica Italiana (Bologna, Oktober 1907) kurz illustriert; der erste wurde auch bei einer Sitzung (15̌. April 1907) der Societáa Milanesi di Medicina e Biologia mitgeteilt. 
Ich glaube nicht, die gestellte Diagnose (tumorbildende Ostitis fibrosa) diskutieren zu müssen; dieselbe erhellt genügend aus den Krankengeschichten and aus den Befunden, wie in bezug auf den ersten Fall Prof. Muscatello (Universität Pavia) erkannte, welcher die entsprechenden Präparate untersuchte.

Obwohl die mikroanatomische Untersuchung in beiden Fällen auf den einzigen Teil des kranken Knochens beschränkt werden musste, welcher mir durch den operativen Eingriff zur Verfügung gestellt wurde, schienen mir beide Beobachtungen einer Illustration wert, und ich wurde dadurch veranlasst, bei denselben und in der Literatur die Frage nach der pathologischen Bedeutung dieser sonderbaren Kuochenaffektionen zu erforschen.

Ebenso wie die anderen bisher bekannten Fälle von umschriebener tumorbildender Ostitis fibrosa, zeigen meine Fälle eine sehr grosse Aehnlichkeit mit dem von Bergmanns Schülern und besonders ron Fürte, Schlange, König und Koch beschriebenen sogenannten cystischen Chondrofibrom der langen Knochen. Dieser als Tumor angesehene Prozess befällt mit Vorliebe die Metaphysen der langen Knochen und besonders die subtrochanterische Gegend des Femurs, äussert sich durch eine Verdickung und eine progressive Verkrïmmung des Knochens, and verursacht ofters spontane Frakturen; die Geschwulst besteht aus faserigem, meistens zellenreichem, Knorpelinseln enthaltendem Gewebe, und stellenweise ,aussehliesslich aus derben Fasergewebe, welches am meisteu dem Faserknorpel gleicht"1); gewöbnlich beobachtet man dałei eine Cystenbildung.

Die klinische wie anatomische Aehnlichkeit ist eine so grosse, dass man mit Recht die zwei Formen als identisch ansprechen kann.

Während mehrere Autoren, wie Glimm, Lexer, Bockenheimer, Gaugele sich darauf beschränken, aus diesem Vergleiche eine allgemeine Schlussfolgerung zu ziehen, in dem Sinne, dass manche Formen von gutartigen Knochencysten, welche in früherer Zeit auf eine Erweichung ron Tumoren zurückgeführt wurden, höchstwahrscheinlich einer lokalen Ostitis zuzuschreiben sind, vereinigt Tietze obne weiteres die als Chondrofibrome boschriebenen Fälle unter dem einheitlichen Begriffe der cystischen Chondrodystrophic im Simne von v. Mikulicz' und v. Mikulicz selbst waren ohne Zweifel, als er die Grundzüge seines Bildes feststellte, dio Cystenexemplare gegeuwärtig, welche von der Bergmannschen Schule untersucht und als neoplastisch angesprochen worden waren.

Dass die Identifizierung eine durchaus ungezwungene ist, kann eine schematische Synopsis der Fälle beweisen, welche das Femur allein oder

1) Körte, angeführt von Tietze. 
zusammen mit der Tibia betreffen, wie sie in nebenstehender Tabelle II zu sehen ist.

Offenbar liegt uns eine und dieselbe Erkrankung vor, welche in verschiedener Weise gedeutet wurde. Nun fragt es sich, welche Deutung die befriedigendste ist?

Ich glaube von den Deutungen von v. Mikulicz und von Beck absehen zu dürfen; der erste hat nämlich keine pathogenetische Theorie aufgestellt, sondern anscheinend vielmehr das Gesamtbild der Affektion bestimmen wollen; während er demselben die Bedeutung einer neoplastischen Affektion abspricht, bezeichnet er ihre vermutliche Natur in einer durchaus unbestimmten Weise ("Wachstumsstörung Osteodystrophie"). Beck hat das Verdienst, die möglichen Beziebungen zwischen der Rachitis und den Knochencysten betont zu haben, Beziehungen, welche in gewissen Fällen nicht zı leugnen sind, jedoch nie nötig und destoweniger direkt sind. Er ordnet die entzündliche Knochenresorption, von welcher die Cyste herstammt, einer akzidentellen Wucherung der metaphysären rachitischen Knorpelreste unter. Hier fragt es sich aber: welcher Natur ist diese Wucherung? und diese Frage lässt auch Beck ungelöst.

Die Streitfrage ist doch im Grunde die folgende: handelt es sich um einen entzïndlichen oder um einen blastomatösen Vorgang?

Offenbar hängt das heutige Vorwiegen der entzündlichen Lehre in erster Linie von dem grossen Anklang ab, welchen die Anschauungen von v. Recklinghausen in bezug auf die diffusen Formen der Ostitis fibrosa fanden. Jedoch können die Betrachtungen, welche den hervorragenden Pathologen dazu veranlassten, die genannten Formen als entzündlicher Natur anzusprechen, nicht obne weiteres auf die umschriebene Varietät der tumorbildenden Ostitis fibrosa bezogen werdell. v. Recklinghausen nimmt an, dass die Skelettsegmente, welche am meisten durch mechanische Zug- und Druckwirkungen in Anspruch genommen werden (dieselben entsprechen bei den Röhrenknochen den Punkten, wo die komplete Knochensubstanz die grösste Dicke apnimmt), der Sitz von habituellen oder sich wiederholenden Kongestionen werden, und dass letztere infolge einer besonderen Reizbarkeit des Substrates zu einer entzündlichen Wucherung des Markes und somit zu seiner fibrösen Umwandlung, zur Bildung von osteoidem Gewebe führen. Kurz, es würde sich um eine chronische, produktive Entzündung, ähnlich den vaskulären Cirrhosen der Eingeweide und der Elephantiasis der Weichteile handeln, "welche von einem Ulcus chronicum ausgehen und unter venöser Stauung verlaufen." Wir haben nun absichtlich darauf hingewiesen, dass die jugendliche umschriebene, tumorbildende Ostitis fibrosa ursprünglich nicht den Diaphysenkörper, d. h. die Knochenteile befällt, welche nach v. Recklinghausen den grössten und konstantesten mechanischen Reizen ausgesetzt sind, 


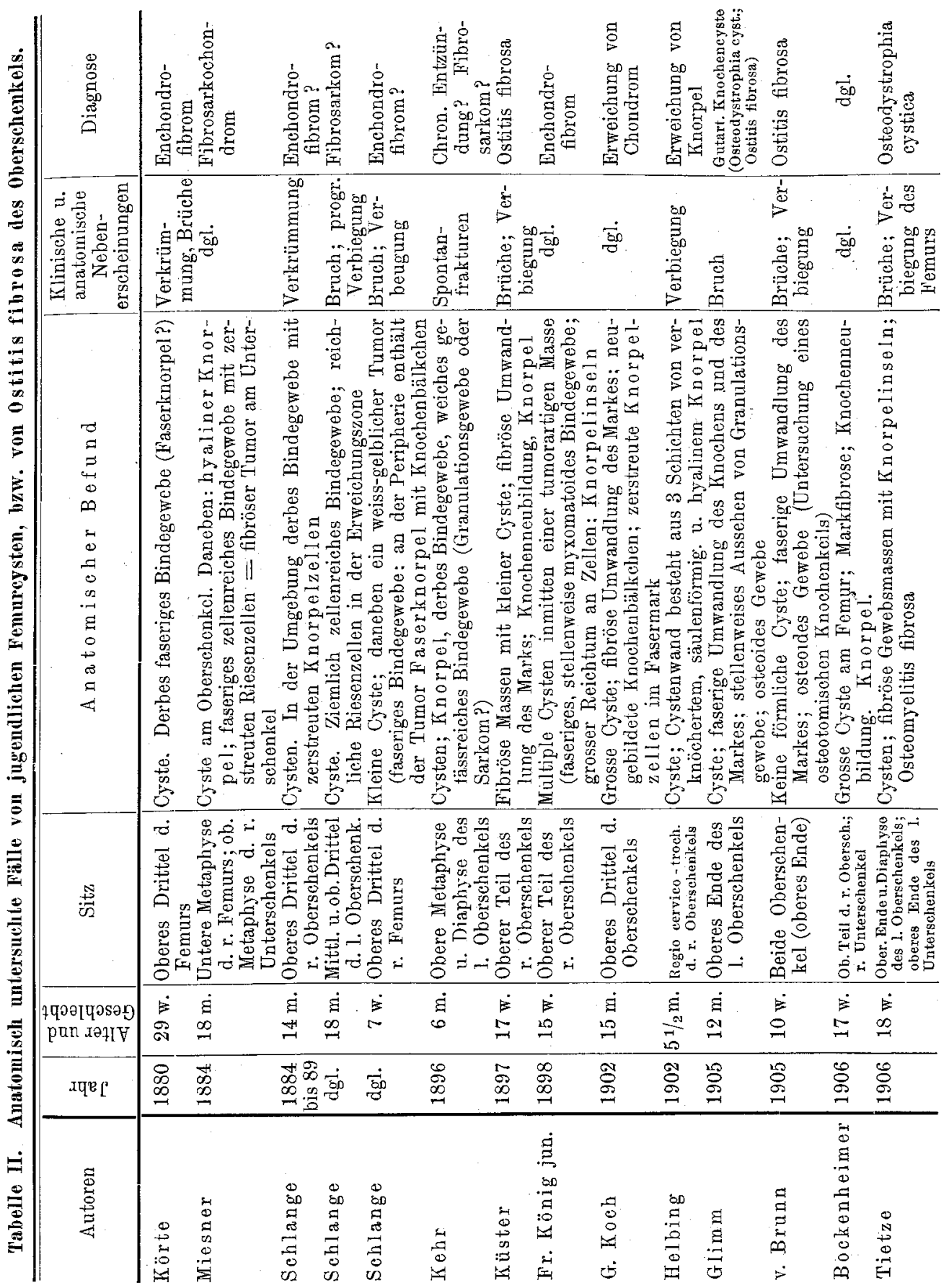


sondern gewöhnlich in den metaphysären Zonen beginnt. Es ist also der erste Sitz ein anderer, und vermutlich auch der Entstehungsmechanismus ein anderer. Es fragt sich nämlich, ob das anfängliche Trauma, welches so oft, ja sogar fast konstant in den Geschichten der Fälle von umschriebener Ostitis fibrosa berichtet wird, als ein Aequivalent der kontinuierlichen mechanischen Frakturen, der danernden Reizungen gelten kann, welche von v. Recklinghausen zugezogen werden, insbesondere wo das genannte Trauma doch, mit Ausnahme der wenigen Fälle, wo Rachitis mitwirkt, anf Knochen wirkt, welche nicht infolge von allgemeinen Dyskrasien, von physiologischer Osteoporosis eine besondere Neigung oder Prädisposition dazu haben, wie diejenigen älterer Leute in dem gesagten Sinne zu erkranken. Das kann man, wenn nicht direkt leugnen, doch wenigstens sehr bezweifeln. Man muss übrigens bedenken, dass sehr oft das Trauma in der Geschichte dieser Erkrankung aus dem einzigen Gruude hervortritt, dass es eine Fraktur herbeigefübrt hat, und bei seiner babituellen Unbedeutsamkeit muss man annehmen, dass das Trauma deshalb eine Fraktur hat bedingen können, weil die Osteopathie boreits seit längerer Zeit bestand. Und wer kann in den Fällen, wo keine frühzeitigen Frakturen stattfinden, behaupten, dass das Trauma dem Beginne des Prozesses ontsprochen hat? Und wenn wir das auch annehmen wollten, können wir aus der Wahrnebmung dieser Ursache Schlussfolgerungen über die Natur des Prozesses ziehen, während bekanntlich die externen Traumata, die mechanischen Reize, die chronischen Reizungen, ebenso die Entstehung bzw. die Lokalisation und die Entwicklung einer Entzündung wie einer Geschwulst hervorrufen können?

Andererseits fragt es sich, welche plausible Ursache für einen entzündlichen Prozess angenommen werden kamı. Jedenfalls kein infektiöses Moment, welches mit genügender Konstanz aus der Anamnese hervorgeht oder durch bakteriologische ${ }^{1}$ ) Untersuchungen als vorhanden bewiesen werden kann; auch kein toxisches Moment (wenn man von den auf rachitischem Boden entwickelten Formen absieht); kurz, keines der gewöhnlichen Entzündung anregenden Momente.

Die Gesamtevolution und die klinischen Erscheinungen des Prozesses sprechen keineswegs für eine entzündlicbe Natur desselben. In dieser Hinsicht ist die besondere Bedeutung, welche den Schmerzen zugeschrieben wird, nicht gerechtfertigt; dieselben stellen ein ganz gewöhnliches Symptom dar, und scheinen nur von dauernder und progressiver Dehnung, von der exzentrischen Expansion des kranken Knochensegmentes abzuhängen, welche die endosteale Umbildung bedingt.

1) Die von $P f_{\theta}$ iffer in seinen Fällen ausgeführten Untersuchungen fielen negativ aus. 
Letztere erscheint bei der chirurgischen Exploration meistens als eine vom Knochen glatt unterschiedene bzw. getrennte Masse, sozusagen als eine vollständige Bildung, als eine - wenn ich den Ausdruck anwenden darf parasitäre Inklusion, welche sich zu den Knochen ebenso verhält wie die echten Tumoren zu deu umgebenden Geweben.

Auch unter dem histologischen Gesichtspunkte haben rlie ersten Beobachter den Prozess ohne weiteres als eine Geschwulst angesprochen und dieselbe meistens als ein Chondrofibrom diagnostiziert. Später ist auch den anderen Autoren, welche diese Deutung bezweifelten oder direkt ablehnten, dies tumorähnliche Aussehen des krankhaften Gewebes nicht entgangen. So scheint Orth, auf (Hrund der Resultate der Untersuchungen von Behrs Fall und besonders des aussergewöhnlichen Reichtums an zellularen Elementen, daza zu neigen, den Prozess, obwohl nicht ohne Vorbehalt, als ein Fibrosarkom anzusprechen.

Ponfick und Tietze') geben bei dem Falle dieses $Z_{\text {weiten } z u \text {, dass }}$ mikroskopisch die fibrösen Massen des Prozesses eine grosse Aehnlichkeit mit einem Fibrosarkom aufweisen.

Bei einer Durchsicht der Berichte über die Befunde fast aller Fälle findet man ein an Kernen sehr reiches Bindegewebe angegeben, welches von einigen Autoren mit dem Granulationsgewebe, von anderen mit dem Sarkom verglichen wird.

Die Einsprengung von Riesenzellen zwischen dem Gewebe bestätigt, obwohl sie nicht absolut pathognomonisch für gewisse Knochensarkome ist, zusammen mit anderen Charakteren und Betrachtungen die Vermutung einer grossen Affinität des Prozesses mit den sarkomatösen Neubildungen.

Wir müssen jedoch noch die Tatsache betonen, dass bei der jugendlichen umschriebenen tumorbildenden Ostitis fibrosa und bei den vermuteten Tumoren aus älteren Beobachtungen, welcbe jedoch jener vollständig entsprechen, das riesenzellensarkomatöse Element nicht so rein individualisiert erscheint wie bei der diffusen v. Recklinghausenschen Krankheit. Es werden stets zerstreute Riesenzellen erwähnt, die auch in einem von meinen Fällen vorkommen, nie jedoch ein Komplex von mikro- und makroskopischen Erscheinungen hervorgehoben, auf deren Grund man von eigentlichen bestimmten riesenzellensarkomatösen Bildungen sprechen darf. Ausserdem scheint es, wie gesagt, dass die Anwesenheit von echten Riesenzellensarkomen stellenweise mit der progressiven und diffusiven Neigung der Osteopathie einhergeht. Nichtsdestoweniger muss man zugeben, dass die Unschlüssigkeit der Autoren bei ihrer Beurteilung und Deutung der Natur des Prozesses vollständig durch die Ergebnisse der histologischen Untersuchung gerechtfertigt ist. Wenn die tumorähnlichen Massen Blastome

1) a. a. 0 . 
sind, wie müssen wir das Faserwerk deuten? Bildet dasselbe einen integrierenden Teil der Geschwulst, indem er sozusagen Auslâufer desselben darstellt, welche in die Umgebung strahlen, um die Markräume zu invadieren, oder bildet es eine accessorische Produktion anderer Natur? Und kann man dieser zweiten Annahme beistimmen, wo man beobachtet, dass Faserwerk und Geschwulst ineinander übergehen, in den meisten Făllen ohne eine erkennbare Grenze?

Tietze ist der Annahme einer neoplastischen Affektion durch die Verschiedenheit, bzw. Nichtübereinstimmung zwischen ihrer klinischen Gutartigkeit und der zerstörenden und invasiven Neigung, welche sie in loco aufweist, abgeneigt.

Nach diesem Autor müsste man, wenn man die Neubildung als einen Tumor ansehen wollte, dieselbe, da sie nie glatt umschrieben gefunden wird, sondern die Markräume infiltriert, das Mark zerstört und stellenweise die Kortikalis arrodiert und zerstört, als eine äusserst bösartige Geschwulst ansprechen, vielleicht bösartiger als die echten zentralen Sarkome, "welche doch bis zu einem gewissen Grade ein geschlossenes Ganze bleiben, das sich in seinem Gefüge schon makroskodisch von der Nachbarschaft trennen lässt." Dagegen könnte man einwenden, dass eine scharfe makroskopische Abgrenzung der Neabildung überhaupt nicht immer fehlt. Sie schien, nach der Beschreibung dieses Autors, im Tietzeschen Falle selbst vorhanden zu sein; sie war ohne $Z$ weifel in unseren Fällen vorhanden, wo die Geschwulstmassen durch Farbe, Homogenität und Konsistenz scharf von der spongiösen Knochensubstanz unterschieden waren, worin sie fast eingebettet waren. Was das diffus infiltrierende Verhalten in loco anbetrifft, welche in offenbarem Gegensatz oder Widerspruch zur klinischen Gutartigkeit des vermuteten Tumors steht, würde wohl die Annahme einer ausschliesslich örtlichen Bösartigkeit der Geschwulst, vergleichbar mit derjenigen von gewissen Hautepitheliomen, zur Erklärung genügen; um so mehr als man Beispiele von derselben auch bei Bindegewebstumoren und besonders bei spindelzelligen Sarkomen und bei Fibrosarkomen findet.

Das wäre jedoch zu bequem. Besser ist es nach meiner Ansicht, sich auf die anatomisch - pathologische Grundauffassung der v. Recklinghausenschen Krankheit zu beziehen, welche wir am Anfang dieser Arbeit erwähnt haben. Wir sehen so, dass die Infiltration des Knochens von Seiten des Tumors stellenweise eine nur anscheinende sein und dieser Anschein von der Unmöglichkeit abhängen kann, bei den eingehenden anatomischen Untersuchungen die zwei Elemente des Prozesses von einander zu scheiden bzw. zu dissociieren: Osteomyelitis fibrosa und tumorartige Bildung.

Wenn letztere, was am häufigsten der Fall ist, die Struktur der 
Fibrome oder der Fibrosarkome hat, dann scheint sie sich direkt mit dem Bindegewebe fortzusetzen, welches die umstehenden offenen Markräume besetzt. Dadurch wird der Anschein einer Diffusion des Neoplasmas in den Naschen des Knochengerüstes hervorgerufen.

Ich muss zwar zugeben, dass dieser Anschein auch die Annahme rechtfertigen kann, dass die Tumoren infolge einer Verdichtung des Fasermarkes an den Stellen entstehen, wo der Kuochen grosse Zerstörungen erlitten hat. Bei der mikroskopischen Untersuchung findet man nämlich im Fasermark und in der Neoplasie eine gegenseitige Kontinuität und Identität der morphologischen Bestandteile, weshalb es logisch erscheinen könnte, beiden die gleiche Bedeutung und die gleiche Herkunft zuzuschreiben. Das ist aber nicht möglich, wenn die Neoplasie eine ganz verschiedene Struktur und Konstitution aufweist, und wenn, wie im zweiten unserer Fälle, das Fasermark scharf umschriebenen Knorpelzonen angrenzt. Hier ist die Komplexität des Prozesses eine offenbare, gleichgiltig welches Abhängigkeitsverhältnis wir zwischen seinen dissociierten morphologischen Elementen annehmen wollen. Da nun die Gleichwertigkeit der zwei homoiomorphen und heteromorphen anatomisch-pathologischen Varietäten des Prozesses zweifelhaft ist, erscheint es mir logisch, anzunehmen, dass sich auch in den Fällen, wo man nur ein Gewebe (faseriges Bindegewebe), zu Massen vereinigt (Fibrome oder Fibrosarkome) und im Knochengerüst infiltriert (Osteomyelitis fibrosa) findet, tatsächlich um zwei verschiedene Alterationen handelt, welche in der histogenetischen Folge sich identifizieren und gleichen, aber dynamisch individualisierbar und wahrscheinlich die eine der anderen untergeordnet sind.

Nur kommt am Ende die Bedentung des bei der jugendlichen tumorbildenden Ostitis fibrosa häufig vorhandenen Knorpelgewebes zur Erwägung. Dasselbe zeigt sich meistens in Form von in der bindegewebigen Hauptmasse enthaltenen Inseln, oder, was seltener der Fall ist, es bildet allein das geschwulstartige Element. Die Anhänger der entzündlichen Lehre haben sich immer bemüht, die Bedeutung dieses Befundes zu vermindern, welcher nach meiner Ansicht eine der befriedigendsten Erklärungen des Prozesses liefert.

Einige Autoren nehmen an, dass der Knorpel ein aus der Epiphysärlinie accidentell versprengtes Gewebe darstellt, und sprechen ihm deshalb jede aktive Teilnahme an Prozess ab. Das ist z. B. die Meinung von $\mathrm{Keh} \mathbf{r}$, welcher bei den in seinem Falle gefundenen Knorpeleinschliessungen deutlich die Verkalkungszone erkannte.

Küster und Bockenheimer schliessen dagegen aus, dass es sich um die Einschliessung von zur Wachstumszone gehörenden Knorpelteilen handle, wie man sie bei rachitischen Knochen beobachtet, und sind der Meinung, 
dass das Knorpelgewebe sich bei der Ostitis fibrosa infolge einer Meta. plasie des Fasermarks bildet.

Andere Autoren glauben dagegen, auf Grund der Analogie mit dem, was Ziegler bei Arthritis deformans beobachtet hat, dass es sich um cine Rekartilaginescenz des Knochens handelt ${ }^{1}$ ).

Tietze äussert seine Meinung hierüber sehr kurz und bündig, wie folgt: „Die Knorpelinseln, welche man in solchen Knochen findet, sind in der Regel nicht zufällig in das Gewebe hineingesprengt, nicht durch Rekartilaginescenz, durch rückläufige Substitution kontinuierlicher Aequivalente entstanden..., sondern der Kuorpel ist an Ort und Stelle gebildet aus einem Bindegewebe, welches das Bestreben hat, sich weiter zu entwickeln und dem die kongenitale Fähigkeit Knochen und Knorpel zu bilden verliehen ist."

Wenn man aber auch die Herstammung der Knorpelinseln von der epiphysären Linie durch zufällige oder rachitische Störung beweist, ist dadurch noch nicht bewiesen, dass dieselben sich bei der Evolution des Prozesses absolut passiv verhalten. Vielmehr finden wir in der Heterotopie eines germinalen Gewebes, wie eine solche gerade die Anwesenheit ron knorpeligen epiphysären Versprengungen inmitten des spongiösen metaphysären Knochens darstellt, die besten Bedingungen für die Entwicklung eines Tumors nach der alten, jedoch besonders in Beziehung auf gewisse Knochentumoren heutzutage noch geltenden Cobnheim schen Lehre geboten.

Und wie könnte man andererseits, wenn man der Anschauung von Köster, von Bockenheimer und von Tietze beistimmen wollte, die Tatsache erklären, dass bei der Ostitis fibrosa der Erwachsenen, wo doch die Erscheinungen einer unvollkommenen Reparation des Knochens von seiten des Fasermarks so ausgedehnt und deutlich sind, nie die Bildung von Knorpel oder von chondroidem Gewebe beobachtet wurde? Man kann doch nicht annehmen, dass die Fähigkeit Knorpel oder chondroides Gewebe zu erzeugen, bei dem Knochen der Erwachsenen fehlt. Gegen diese Annahme würde schon deutlich die Evolution des Kallus bei Frakturen sprechen. Dass der zufällig oder infolge einer früheren Osteopathie versprengte Knorpel eine wesentliche Rolle bei der Entwicklung des Prozesses spielt, haben, wenn auch in verschiedenem Sinne, König und Beck angenommen. Für diese Annahme spricht in indirekter Weise die Tatsache, dass in einigen Fällen die ganze Neubildung das genaue Aussehen des Faserknorpels zeigt, und dass man in anderen Fällen, z. B. im ersten

1) Dieser Ansicht ist auch Wiesner, einer der ersten Beobachter des Pro* zesses und Verteidiger der neoplastischen Lehre. Er glaubt nämlich, dass die chondromatösen Bildungen aus durch Metaplasie vom Knochen herstammenden Knorpelinseln hervorgehen, 
der unseren, deutlich gekapselte Elemente zwischen dem faserigen Bindegewebe, auch entfernt von den echten Knorpelinseln zerstreut findet. In unserem ersten Falle selbst zeigen die Faserknorpelknoten Charaktere, welche in ausgezeichneter Weise mit der Annahme einer germinalen Funktion im Cobnheimschen Sinne zusammenstimmen; es befindet sich besonders einer dabei (s. Fig. 10), welcher durch seinen Sitz und seine Struktur eine besondere Bedeutung im Bildkomplex annimmt. Er befindet sich sehr nabe der Korticalis in einer Zone des untersuchten Stückes, welche genau dem Gipfel der Krümmung des Femurs entspricht, d.h. dem

Figur 10.

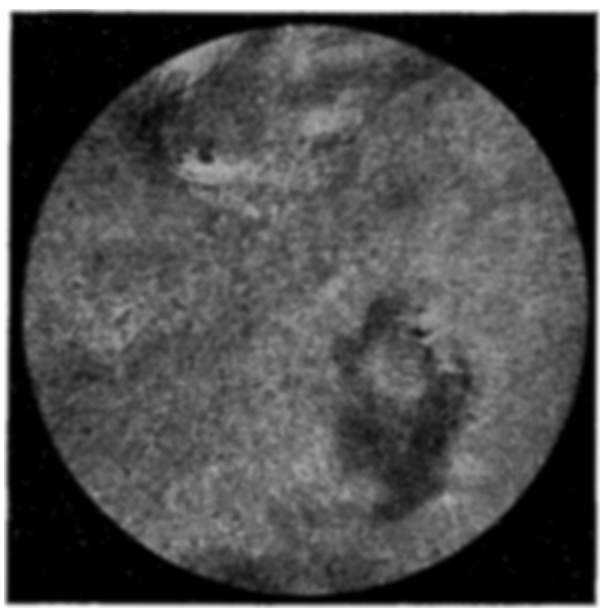

Faserknorpelige Zone (Fall I).

Punkte, wo die Neoplasie anscheinend die grösste Entwicklungstätigkeit entfaltet hat und sozusagen, durch ihre Expansion die Wandung des diaphysären Zylinders ausgedehnt hat. Es sind dort zahlreiche zweikernige Knorpelkapseln vorhanden, welche vermutlich eine gegenwärtige Wucherung der Elemente bezeugen; ausserdem ist der Uebergang von chondroidem zum umgebenden fibrösen Gewebe keineswegs ein direkter; die Kapseln werden allmählich undeutlicher, bis sie verschwinden, die Zellen verlängern sich allmählich bis sie spindelförmig werden, was anscheinend die verschiedenen Phasen der rückläufigen Evolution des Chondroblast in Fibroblast darstellt. Ob eine solche Evolution in der. Tat hier zu Stande kommt, darf ich nicht ohne weiteres behaupten; ein direkter Beweisgrund dazu fehlt mir. Jedenfalls erseheint es nach dem obigen Befunde wahrscheinlieh. Was ihre Möglichkeit prinzipiell 
anbetrifft, bemerke ich, dass sie a priori nicht diskutierbarer ist, als diejenige der Umwandlung des Faserwerks im Knorpel oder der Rekartilaginescenz des Knochens.

Ein allmähliches Uebertreten des Knorpels in die zellenreiche Geschwulst ist auch in dem von Tietze berichteten Königschen Befunde erwähnt; letzterer unterscheidet sich jedoch von meinem dadurch, dass der Knorpel byalin war und an einigen Punkten desselben die Stadien der enchondralen Verknöcherung bis zur vorläufigen Verkalkung erkennbar waren $^{1}$ ).

Im zweiten unserer Fälle bestanden die Zonen mit tumorartigem Aussehen durchwegs aus hyalinem Knorpel, welcher hier und da durch ein bindegewebiges Stroma in kleine Abteilungen geteilt war. Wenn wir auch annehmen wollen, dass die leichte, vorangegangene Rachitis die Entwicklung dieser knorpeligen Bildungen und die Zerstreuung der Keime derselben in der Metaphysis veranlasst habe, hindert uns nichts daran, sie als echte Chondrome anzusprechen. Neben letzteren, aber ganz unabhängig und unterschieden von der Knorpelwucherung, finden wir die Osteomyelitis fibrosa; das an einigen Punkten in der Nähe der Knorpelzonen verdichtete Faserwerk setzt sich nur mit den faserigen Septa fort, welche die genannten Zonen durchschneiden.

Hier ist kein fibröser Teil vorhanden, welcher als integraler Bestandteil zum Tumor gehört und sich mit den Knorpelinseln fortsetzt oder von denselben auszustrahlen scheint; wir finden aber auch nicht das geringste Zeichen, welches darauf hinweisen könnte, dass der Knorpel von einer Umwandlung des Fasermarkes berrührt, von welchen er fast immer durch die Reste alter; durch die Osteoklasten arrodierter Bälkchen getrennt ist (Fig. 11).

Der Einwand von Glimm²) und von Tietze, dass die Veränderungen der jugendlichen cystischen Osteodystrophie fast nie eine genügende Analogie mit den echten Chondromen aufweisen, und dass dieselben sich besonders vom Bilde des Virchowschen typischen Falles entfernen, ist jedenfalls begründet, jedoch nicht entscheidend. Es ist zwar wabr, dass in dem besagten Falle (Cyste des Humeruskopfes bei einer 56jährigen Frau) keine Deformität des Knochens und keine Verdünmung der Kortikalis

1) Könjg bemerkt noch (wodurch die Analogie der Befunde besser bestätigt wird): „Auffallend ist der stellenweise grosse Reichtum an Nerven, spindelförmige und mehr runde Zellen sind an manchen Stellen so reichlich vorhanden, dass man hier histologisch die Diagnose des Sarkoms machen konnte. Dann lommen freilich wieder ganze Strecken mit spärlichen Fibromzellen und an wieder anderen findet sich der Typus des myxomatösen Gewebes."

2) a. a. 0 , 
zu sehen war, und man zahlreiche kleine aus Netzknorpel gebildete und von vollständig normaler spongiöser Knochensubstanz umgebene Tumoren vorfand ${ }^{1}$ ). Man will aber nicht behaupten, dass es sich um mit jenen

Figur 11.

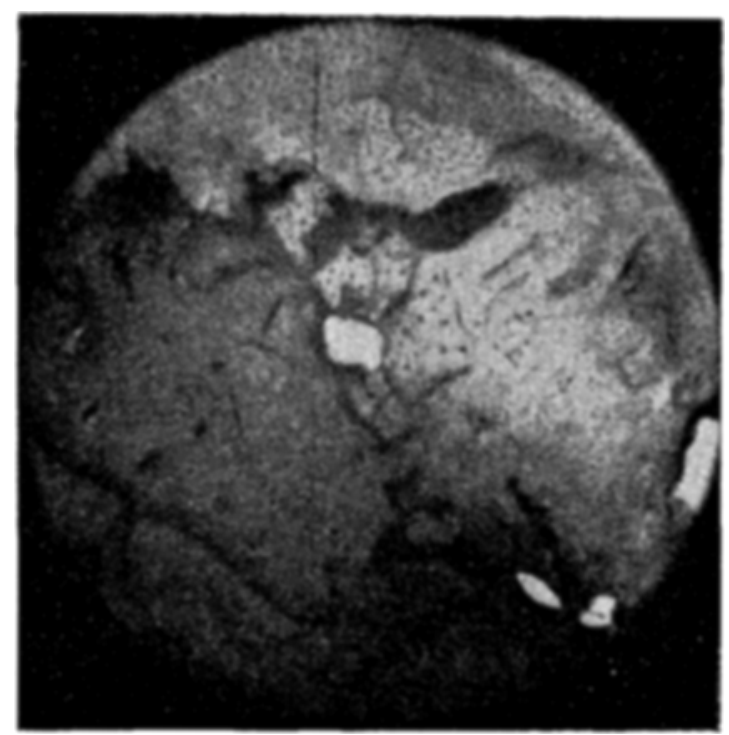

Knorpel- und Fasermark, getrennt durch Reste von in Resorption begriffenen Bälkchen (Fall lI).

identische Tumoren handelt. Es handelt sich höchstwahrscheinlich um eine komplette pathologische Form, zu welcher ein neoplastischer, nach

1) Lexer spricht seinem Fall jede Verwandtschaft mit der Ostitis fibrosa $a b$, und zwar deshalb, weil bei demselben die metaphysären Knorpelinseln, welche ein vermutliches Zoichen einer grösseren präexistierenden chondromatösen Masse darstellen, die durch ihr Schmelzen die Cyste erzeugt bätte, von normalem Knochengewebe umgeben waren. Er bemerkt jedoch: Einzelstellen der Wandung hätten mit den neugebildeten Knochenbälkchen, welche hier und da Knorpelzellen enthielten, mit den organisierten Blutmassen in den Markräumen und der stellenweise kräftigeren Bindegewebsentwicklung in ihnen leicht zu der Annahme von Ostitis fibrosa mit Cystenbildung geführt (Lexer, a. a. 0.).

Dies beweist wenigstens, dass die Anwesenheit eines Tumors - wenn wir Lexers Schlussätze unterschreiben wollen - oder einer Cyste neoplastischen Ursprungs im Knochen der Ostitis fibrosa ähnliche Nachbarschaftsveränderung hervorrufen kann. 
meiner Ansicht áls primär anzusehender Bestandteil, und ein sekundärer, reaktiver Bestandteil beitragen, welch letzterer vielleicht besser als wie ein entzündlicher, wie ein involutiver bezeichnet werden kann. Das ist die sogenannte Osteomyelitis fibrosa, welche jedoch nicht eine direkte Reaktion des Knochengewebes gegen den primären Prozess, sondern eine mittelbare Reaktion darstellen würde. Die Gesehwulst, wie wir sie anch auffassen mögen, ruft, aus Gründen, dic nicht ganz klar sind und bei welchen auch die wiederholten Traumen, die Rachitis und vielleicht eine besondere Verletzbarkeit des im Wachstum begriffenen Knochens eine Rolle mitspielen könnten, in ihrer Umgebung, und zwar in einer ziemlich ausgedehnten, oft nicht im Verhältnis zu dem Volumen des Tumors stehenden Zone Erscheinungen der halisteretischen Osteoporosis hervor. Letzteren folgt, immer von denselben vielmehr vermuteten als gekaunteu Ursachen abbängig, eine Fibrosis des Markes, vorwiegend mit der Bedeutung einer Hyperplasie ex racuo, und die Bildung von osteoiden Bälkchen von seiten des Fasermarkes, mit der Bedeutung eines Reparationsversuches.

Was das blastomatöse Element ambetrifft, so besteht dasselbe jedenfalls sehr selten, wie in einem unserer Fälle, aus echten Chondromen. In den bisher berichteten Befunden findet man neben einer konstanten Identität der histologischen Bestandteile (faseriges Bindegewebe, Riesenzellen, Knorpel), einen bedeutenden Polymorphismus, welcher ausschliesslich die Art der Kombination der Bestandteile selbst anbetrifft. Manchmal ist Knorpel in überwiegendem Masse vorhanden und die Geschwulstnähert sich in ihrer Struktur einem Chondrom; ofter hat das fibromatöse oder fibrosarkomatöse Gewebe die Ueberhand; seltener, wie im Falle von Pfeiffer und in demjenigen von Bötticher-Mönckeberg, kommt es zum histologischen Typus des Riesenzellensarkoms ${ }^{1}$ ).

Auf Grund aller bisher dargelegten Betrachtungen bin ich der Ansicht, dass man, solange in der Pathologie die Unterscheidung zwischen entzündlichen und echten Neoplasien (Blastome) bestehen bzw. gelten wird, die jugendliche umschriebene Form der tumorbildenden Ostitis fibrosa als eine Affektion vorwiegend geschwulstigen Ursprunges und geschwulstiger Natur ansprechen muss.

Die Tumoren, welche sie charakterisieren, müsste man, wenn man sic nach ihrer häufigsten Zusammensetzungsart benennen wollte, als Chondrofibrosarkome benennen.

Diese Bezeichnung bzw. Deutung kann als ein doktrinaler Rückschritt

1) Nach Schlange ,begründet das Schwanken zwischen bindegewebigen, kmorpeligen und giganto-zellular-sarkomatösen Formen keine prinzipiellen Unterschiede. . . zumal in klinischer Beziehung die Uebereinstimmung der Kranliheits. erscheinungen zu einem auffallend einheitlichen Krankheitsbilde fïhren." 
scheinen, besonders, wenn man die Frage in bezug auf den Ursprung der Knochencysten betrachtet.

Heutzutage neigt man nämlich dazu, diese letzteren als den Ausgang eines entzündlichen Prozesses des Knochens zu betrachten, und zwar deshalb, weil man ihre häufigere Entstehung aus dieser lokalen Erkrankung feststellte, welche auf eine umschriebene Varietät der v. Recklinghausenschen Krankheit bezogen wurden. Bei der Auffassung dieses letzteren musste jedoch, wie wir gesehen haben, die Ostitis fibrosa den chronischen, interstitiellen Entzündungen gleichgestellt werden, welche eine Wucherung und eine Verdichtung des Bindegewebes, zum Schaden des Parenchyms, mit einem Worte eine Cirrhose, hervorrufen. Diese Prozesse bedingen nur dann eine Cystenbildung, wenn sie in der Struktur des befallenen Organs besondere prädisponierte Bildungen treffen, aus welchen sich die Cyste entwickeln kann. Es handelt sich also um Retentionscysten echt mechanischen Ursprungs. An und fïr sich neigt das hyperplastische entzündliche Gewebe, falls es entartet, vielmehr dazu, sich zusammenzuziehen, zu sklerosieren, eventuell zu verkalken, als zu verschmelzen. Auch diese Betrachtung würde somit gegen die entzündlichneoplastische Auffassung der faserigen Gewebsmassen bei der Ostitis fibrosa mit Tumoren- und Cystenbildung sprechen. Denn, wenn die Knochenrarefaktion und besonders die lakunäre Resorption in hohem Nasse an der Bildung und dem Wachstum der Knochenhöhley sich beteiligen, so kam nicht in Abrede gestellt werden, dass sich die Cysten immer oder fast immer in unmittelbarer Nähe, und zuweilen inmitten der Nassen neoplastischen Aussehens und offenbar auf Kosten derselben bilden.

Nun gehören die involutiven Vorgänge, welche zur Erweichung und zur Verschmelzung führen, wenn nicht ansschliesslich, doch wenigstens vorwiegend zu den geschwulstigen Geweben; die Labilität und die Neigung zur Entartung kommen den Zellelementen der Blastome zu; sie stellen nämlicb, znsammen mit der besonderen Wucherungstätigkeit, jenen Komplex von progressiven und regressiven Tätigkeiten dar, welches als Anaplasie bezeichnet wurde.

Weun man dann dem hämorrhagischen Momente einen Platz in der Entwicklung der Cysten einräumen wollte, kann daranf hingewiesen werden, dass Blutungen viel häufiger inmitten der Tumoren als der Produkte der chronischen entzündlichen Prozesse - abgesehen von den spezifischen Granulationen - zu beobachten sind.

Die cystische Evolution der hämorrhagische Herde und das nachfolgenue Wachstum der Cyste erklärt sich leichter in einer Geschwulst, d. h. in einem atypischen Gewebe mit geringer Reaktions- und Widerstandsfähigkeit, als in einer entzündlichen Neubildung, welche im Falle der Ostitis fibrosa fast ein narbiges Produkt darstellen mïsste. 
Mich nochmals auf die von mir angenommene Definition des anatomisch-pathologischen Begriffes der v. Recklinghausenschen Kuochenkrankbeit beziehend, fühle ich mich logisch dazu geneigt, auf die typische, diffuse und dem vorgeschrittenen Alter eigentümliche Form die Schlusssätze auszudehnen, zu welchen ich hinsichtlich der jugendlichen umschriebenen Formen gekommen bin.

Ich bin somit der Ansicht, dass auch bei der diffusen Form die geschwulstartigen Bildungen, d. h. die Fibrome (Fibrosarkome?) und die Riesenzellensarkome echte Tumoren darstellen; dass die Markfibrose, deren entzüudliche Natur nicht klar bewiesen ist, nicht allein das Grundwesen der Krankheit, sondern vielmehr eine indirekte reaktive Erscheinung darstellt, welche eine besondere Extension und Intensität infolge der besonderen Reiztbarkeit des osteomalacischen Bodens annimmt, auf welchen sich das selbständige blastomatöse Element einpflanzt ${ }^{1}$ ).

Meine Anschauungen stimmen also in Bezug auf die Bedeutung der Riesenzellensarkome mit denjenigen von v. Haberer überein, und scheinen mir durch die im ersten Teil dieser Arbeit erwähnten Betrachtungen über das Verhalten solcher Bildungen bestätigt. Obwohl diese Schlussfolgerungen etwas gewagt erscheinen können, glaube ich nicht, dass man sie als vollständig unbegründet betrachten kann, da sie doch, im Grunde genommen, die von v. Recklingh a usen selbst angezeigte Analogie zwischen der tumorbildenden Ostitis fibrosa und der osteoplastischen Karzinose bestätigen. Bei letzterer unter der Wirkung desselben prädisponierenden Momentes (senile Osteodystrophieen) ruft die metastatische Zerstreuung und Infiltration ron epithelialen Knoten in den Skelettgeweben gleiche relative Erscheinungen (Markfibrose, osteoide Bildungen) hervor, obwohl in verschiedenem Grade und mit verschiedener Ausdelmung, und erzeugt ein ähnliches klinisches Bild.

Augenscheinlich ist auch bei der $v$. Recklinghausenschen Knochenkrankheit die Osteomyelitis fibrosa oder besser der Komplex von involutiven und reparatorischen Vorgängen des Knochenmarkes als eine akzessorische Erscheinung anzusehen, welcher die Entwicklung der Tumoren zwar eine Gelegenheit, jedoch keine genügende Ursache liefert, sich zu entfalten. Somit ist nicht ausgeschlossen, dass dieselbe, wie bei der Pagetschen Krankheit, auch mit einem noch nicht bestimmten und wahrscheinlich nicht immer identischen dyskrasischen Faktor in Zusammenhang stehe. Sei der-

1) Der Ausdruck, tumorbildende Ostitis fibrosa, welcher ich bisher ans Rücksicht gegen den Gebrauch und gegen die Autorität von v. Recklinghausen angewendet habe, entspricht deshalb nicht genau meiner Auffassung der Osteopathie. Diese könnte man besser mit dem Ausdruck "Knochentumoren mit oste0plastischer Markfibrose" bezeichnen. 
selbe eine späte Erbsyphilis oder eine endogene (Gicht, Arthritismus) oder exogene (Mineralsüuren) Intoxikation, wie neuerdings gerade für die Pagetsche Form angenommen wurde, er scheint vermittels des Gefässystems zu wirken, indem er eine diffuse Arterioklerose hervorruft oder befördert. Letztere scheint ihrerseits die physiologische Knochendystrophie des vorgeschrittenen Alters zu erhöhen, und bis fast zu einer echten Osteomalacie zu treiben. So wird der Boden für die reaktiven osteoplastischen Vorgänge geschaffen; diese können ohne eine deutliche intermittierende Ursache entstehen, wie es bei der Pagetschen Krankheit der Fall ist, oder die Tumorenbildung begleiten, und dies wäre nach meiner Ansicht bei der v. Recklinghausenschen Krankheit der Fall.

Ich habe hier vollständig von der therapeutischen Frage abgesehen, weil ich auf diesem Gebiete nichts Neues hätte hervorbringen können. In unseren Fällen wurden die allein zu orthopädischen $Z$ wecken vorgenommenen palliativen Eingriffe sehr gut ertragen, von einer ziemlich prompten Konsolidation mit guten unmittelbaren Resultaten gefolgt ${ }^{1}$ ).

\section{Literaturverzeichuis.}

Beck, American journal of med. sciences. 1901. Beck, Arch. f. klin. Chir. Bd. 70. H. 3. 1903. Bockenheimer, Arch. f. klin. Chir. Bd. 81. T. 2. 1906.

Bockenheimer, Ostitis deformans fibrosa der Schädel- und Gesichtsknochen. Arch. f. klin. Chir. Bd. 85. H. 2. 1908.

Braun, Beitr. zur klin. Chir. Bd. 52. H. 2. 1906.

Cagnetto, Osteo - arthropatie hypertrophiante pneumique von Marie. Rivista veneta di scienze mediche. Agosto 1906.

Gaugele, Fortschritte auf dem Gebiete der Röntgenstrahlen. Bd. 9. 1906.

Gaugele, Arch. f. klin. Chir. Bd. 83. H. 4. 1907.

Guaccero, Pagets Knochenkrankheit. Archivio Italiano dj Ortopedia.

Glimm, Deutsche Zeitschr. f. Chir. Bd. 80. S. 476.

v. Haberer, Arch. f. klin. Chir. Bd. 76. H. 3. 1907.

Hart, Beitr. zur path. Anat. u. allgem. Path. Bd. 36. 1904.

Jones und Morgan, Arch. of the Roentgen-ray. 1907.

Lexer, Arch. f. klin. Chir. Bd. 81. T. 2.

Medea et Ba Fan o, Pagets Knochenkrankheit. Il Morgagni. 1906. F. 6. v. Mikulicz, Centralbl. f. Chir. 1904. S. 1898.

Morpurgo, Experimentelle Rachitis und Osteomalazie. Aroh. per le scienze mediche. Bd. 31. No. 1.

1) Ein besonderer Dank soll hier Herrn Prof. Galeazzi ausgesprochen werden, welcher mir in sehr entgegenkommender Weise die zwei Fälle zur Verfügung stellte und in jeder Hinsicht meine Untersuchangen und Forschungen förderte. 
Oettinger und Agasse-Lafont, Pagets Krankheit. Nouv. Lonogx, de la Salpetrière. Mai. 1905.

Pfeiffer, Beitr, z. klin. Chir. Bd. 53. H. 2. 1907.

v. Recklinghausen, Festschrift f. R. Virehow. Berlin. 1891.

Schmieden, Deutsche Zeitschr. f. Chir. Bd. 70. 1903.

Schuchardt, Krankheiten der Knochen. Deutsche Chirurgie. Licf. 28. Stuttgart 1899.

Sonnenburg, Fortschritte auf dem Geb. d. Roentgenstr. Bd. 8. 1905.

Tietze, Beitr. z. kilin. Chir. Bd. 52. H. 2. 1906.

Almerini, Atti della società Milanese di medicina e biologia. Vol.2. F.1. 1907.

Almerini, 14. Congresso della società ortopedica Italiana. Bologna. 1907.

Im übrigen siehe die Arbeiten von v. Haberer und Bockenheimer, welche ein vollständiges Literaturverzeichnis onthaiten.

\section{Erklärung der Figuren anf Tafel XVIr.}

Figur 2. Fall I. Roentgenbild. Vergrösserung und Verbiegung des Oberschenkels.

Figur 3. Fall I. Roentgenbild nach der Behandlung.

Figur 7 . Fall Il. Roentgenbild des oberen Endes des rechten Femur. 


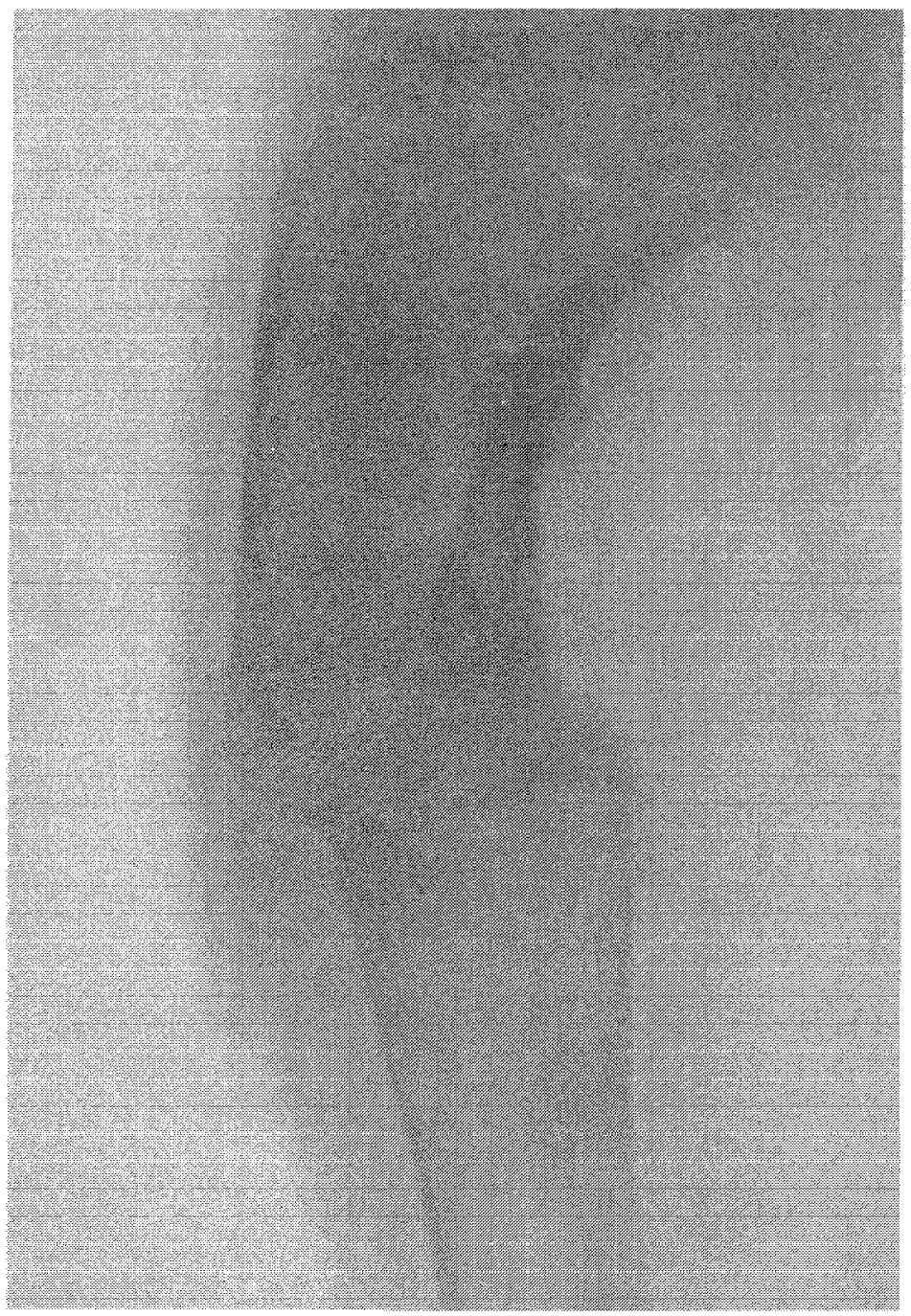

Fig. 3 